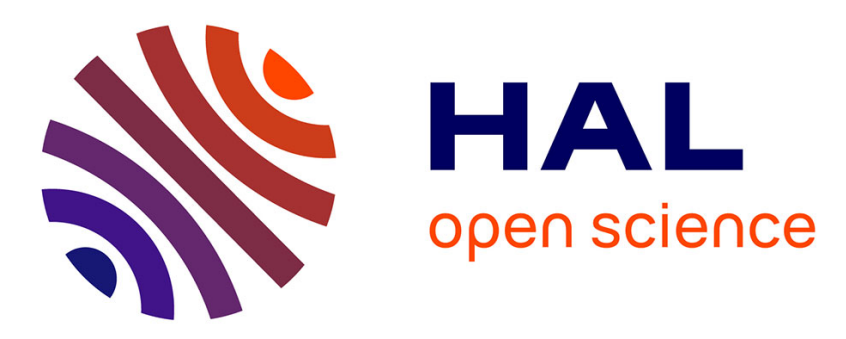

\title{
Aux portes de Strasbourg: une occupation de longue durée à Koenigshoffen, en bordure de la route des Romains
}

\author{
Axelle Murer, Adeline Pichot
}

\section{- To cite this version:}

Axelle Murer, Adeline Pichot. Aux portes de Strasbourg: une occupation de longue durée à Koenigshoffen, en bordure de la route des Romains. Archimède: archéologie et histoire ancienne, 2020, Archimède. Archéologie et histoire ancienne, 8, pp.161-179. 10.47245/archimede.0008.ds3.02 . halshs-03280089

\section{HAL Id: halshs-03280089 \\ https://shs.hal.science/halshs-03280089}

Submitted on 7 Jul 2021

HAL is a multi-disciplinary open access archive for the deposit and dissemination of scientific research documents, whether they are published or not. The documents may come from teaching and research institutions in France or abroad, or from public or private research centers.
L'archive ouverte pluridisciplinaire HAL, est destinée au dépôt et à la diffusion de documents scientifiques de niveau recherche, publiés ou non, émanant des établissements d'enseignement et de recherche français ou étrangers, des laboratoires publics ou privés. 
1 DOSSIER THÉMATIQUE 1

SIUE DEUS SIUE DEA. DÉNOMINATIONS DIVINES DANS LES MONDES GREC ET SÉMITIQUE : UNE APPROCHE PAR LE GENRE

98 DOSSIER THÉMATIQUE 2

TRADITION ET TRANSMISSION DANS L'ANTIQUITÉ : RÉFLEXIONS INTERDISCIPLINAIRES

ACTUALITÉ DE LA RECHERCHE

QUOI DE NEUF Á L'OUEST DE STRASBOURG ? KOENIGSHOFFEN :

ÉTAT DES LIEUX ET DÉCOUVERTES RÉCENTES

159 Michel Humм

Les acteurs de I'archéologie préventive et I'UMR 7044 ArcHiMèdE : une collaboration fructueuse

161 Axelle MURER et Adeline PICHOT

Aux portes de Strasbourg : une occupation de longue durée à Koenigshoffen, en bordure de la route des Romains

180 Marianne BérAud et Adeline PICHOT

Deux nouvelles inscriptions de légionnaires de la légion VIII Augusta

195 VARIA 


\section{AUX PORTES DE STRASBOURG : UNE OCCUPATION DE LONGUE DURÉE À KOENIGSHOFFEN, EN BORDURE DE LA ROUTE DES ROMAINS}

\author{
Axelle MURER \\ Archéologue, Responsable d'opération \\ ANTEA-Archéologie \\ axelle.murer@antea-archeologie.com
}

\author{
Adeline PICHOT \\ Archéologue, Responsable d'opération \\ ANTEA-Archéologie \\ adeline.pichot@antea-archeologie.com
}

\section{RÉSUMÉ}

La fouille réalisée en 2018 au 2, route des Romains sur le site de I'ancien terrain de I'ASPTT de Koenigshoffen a livré une occupation de longue durée comprise entre le Ier siècle de notre ère et le Second Moyen Âge. Des traces d'activités et d'habitats remontant à la fin du XIXe siècle ou au début du $X X$ e siècle ont également été reconnues sur cette opération. Le site, particulièrement malmené par les terrassements préalables à la mise en place de la plaine sportive à la fin des années 1960, a plus particulièrement permis de mettre au jour les limites est de la nécropole dédiée aux soldats des $\mathrm{II}^{\mathrm{e}}$ et VIII légions Augustes, un nouveau quartier de potiers, des restes d'habitats du Bas-Empire

MOTS-CLÉS

Archéologie, funéraire, habitat,

artisanat, agriculture. et médiévaux inédits, ainsi qu'une occupation postérieure à la guerre de 1870, aménagée dans les secteurs non constructibles des fortifications strasbourgeoises.
The excavation carried out in 2018 at 2 route des Romains on the former site of the Koenigshoffen ASPTT sports complex showed a long-term occupation of the site from the 1st century to the late Middle Ages. Traces of activity and settlements dating back to the end of the 19th century or the beginning of the 20th century were also identified during this excavation. The site, particularly affected by earthworks carried out at the end of the 1960s during the building of the ASPTT sports complex, particularly brought to light the eastern boundary of the necropolis for soldiers of the Augustan legions II and VIII, a new potters' district, previously unknown traces of settlements from the Late Roman Empire and the Middle Ages and signs of occupation from after the 1870 Franco-Prussian war in the non-constructible areas of the Strasbourg fortifications.
KEYWORDS

Archaeology, funerary, settlement, craftwork, agriculture. 


\section{PRÉSENTATION SOMMAIRE DU SITE}

\section{CONTEXTE GÉNÉRAL DU SITE}

Cette opération réalisée en 2018 s'inscrit dans le cadre des travaux d'extension de la ligne $F$ du tramway strasbourgeois [1]. Elle fait suite à deux diagnostics archéologiques positifs réalisés en 2013 sur le site de I'ASPTT de Strasbourg-Koenigshoffen, en bordure nord de l'actuelle route des Romains [2]. Les parcelles fouillées sont situées à l'entrée est du faubourg de Koenigshoffen, au pied de I'autoroute A35, sur une ancienne terrasse alluviale dominant la plaine ello-rhénane.

La fouille a été réalisée sur une surface totale de $4943 m^{2}$ comprenant un décapage extensif sur une bande de $35 \mathrm{~m}$ de large, le long de la route des Romains, et deux tranchées de suivi de travaux réalisées sous cette même voie.

Durant cette opération, les archéologues ont malheureusement constaté qu'une partie du site archéologique avait été endommagée lors des terrassements préalables à l'aménagement du terrain de sport, à la fin des années 1960, entraînant un fort arasement ou parfois la disparition de nombreuses substructions telles que des fosses de fondation de bâtiments ou encore des éléments de parcellaire et épargnant les vestiges en creux tels que les caves et les fosses. Ce constat explique l'état parfois lacunaire des occupations successives mises au jour, comme par exemple le peu de vestiges funéraires tels que des sépultures à crémation secondaire en fosse ou encore l'absence de bâtiments liés aux caves exhumées.

Si quelques traces d'une présence durant les époques Néolithique et Protohistorique ont été observées dans le quartier de Koenigshoffen, c'est malgré tout son passé antique qui prédomine avec de nombreuses découvertes archéologiques régulièrement recensées à partir du XIXe siècle dans le cadre de travaux liés au développement de ce faubourg strasbourgeois. Les premières découvertes, fortuites, sont principalement d'ordre funéraire avec des éléments lapidaires, des tombes ou du matériel céramique, et ont eu lieu lors de la pose de canalisations ou de constructions. De 1968 à 1996, de nombreuses opérations de fouilles ont été dirigées par Erwin Kern (SRA Alsace) et ont permis de cerner en partie la structure de l'espace urbain, mais leurs résultats restent mal connus. Depuis, les fouilles préventives menées sur l'emplacement de l'agglomération antique améliorent progressivement la connaissance du tissu urbain. À partir du début des années 2010, de nombreux travaux d'urbanisation, menés afin d'étendre la ligne $\mathrm{F}$ du tramway jusqu'au cœur de l'actuel quartier de Koenigshoffen, ont permis de mettre au jour des vestiges archéologiques très importants, en particulier au nord de la route des Romains.

L'occupation gallo-romaine s'organisait autour de l'axe principal du vicus que constituait le decumanus maximus, aujourd'hui en partie reconnu sur le tracé de la route des Romains et de la rue des Capucins. Cette voie trouvait son origine aux abords du grand camp militaire d'Argentorate et menait à Saverne (Tres Tabernae) puis à Metz (Divodorum). Elle structurait la ville en servant de base à l'établissement d'une trame viaire à peu près orthogonale, en fonction des écueils topographiques existants.

Mis en place au début du Ier siècle de notre ère, sur le tracé d'un probable axe plus ancien, et longé par des monuments funéraires, le decumanus maximus connaît un développement dès la première moitié du II ${ }^{e}$ siècle, notamment avec l'implantation de voiries annexes. Ces réseaux ont permis de faire progresser I'urbanisation du vicus dans des parcelles éloignées de la route des Romains et des secteurs, auparavant dévolus au domaine funéraire. Cette densification de I'habitat se manifeste par la mise en place de structures comme des caves, des bâtiments, des puits ou des latrines se développant perpendiculairement à ces différents cheminements.

Le dynamisme de l'agglomération atteint son apogée au cours du II $\mathrm{I}^{\mathrm{e}}$ siècle. Les vestiges postérieurs à la fin du III e siècle sont moins nombreux, cette période semblant correspondre à un repli de l'agglomération durant le Bas-Empire. 
Dans les derniers travaux qui ont marqué la connaissance du passé antique de Koenigshoffen, la fouille menée par Pascal Flotté (Archéologie Alsace) aux 8-20 route des Romains en 2014 et 2015 est particulièrement remarquable [3]. Parmi les importantes découvertes réalisées figurent plusieurs enclos funéraires avec des stèles, de nombreux éléments mobiliers et des crémations en très bon état de conservation qui permettent d'établir le plan d'un tronçon de la nécropole, en reconnaissant des espaces funéraires distincts. Plusieurs sépultures accompagnées de monuments funéraires alignés le long de la route témoignent du fait que cette nécropole a été en usage entre la première moitié du Ir $^{\text {r }}$ siècle et le début du II ${ }^{e}$ siècle, puis démantelée et remblayée dans la première moitié du II ${ }^{e}$ siècle, au profit de la mise en place de parcelles $\mathrm{d}$ 'habitation [4]. Ces dernières accueillaient plusieurs bâtiments élevés sur vide-sanitaires, répartis de part et d'autre d'une ruelle de desserte (perpendiculaire à la route des Romains), une trentaine de caves, et une trentaine de puits aménagés dans les cours à l'arrière des habitations [5].
Un diagnostic archéologique, conduit en novembre 2017 par Pascal Flotté, à l'emplacement d'une ancienne station-service Total aux 48-58 route des Romains, a livré plusieurs vestiges datés du Ier au III ${ }^{e}$ siècle de notre ère, parmi lesquels une possible voie, plusieurs restes d'habitats matérialisés par des fosses de fondation, ainsi que des témoins de l'urbanisation progressive du quartier de Koenigshoffen au cours du XVIII siècle et du début du XIXe siècle (murs de grès rose et de briques, dont un muni d'un arc de décharge, sol de mortier de chaux). Suite aux résultats de ce diagnostic, une fouille archéologique préventive a été prescrite, mais les résultats n'ont pas encore été communiqués.

Au printemps 2018, une autre fouille préventive a été menée simultanément à celle du 2 , route des Romains par Pascal Flotté (Archéologie Alsace), sur des parcelles adjacentes situées à l'ouest de cette dernière opération, en bordure de l'autoroute et de la voie ferrée, sur une surface de $2520 \mathrm{~m}^{2}$. Les vestiges mis au jour correspondent à la continuité de ce qui a été observé sur le site étudié.

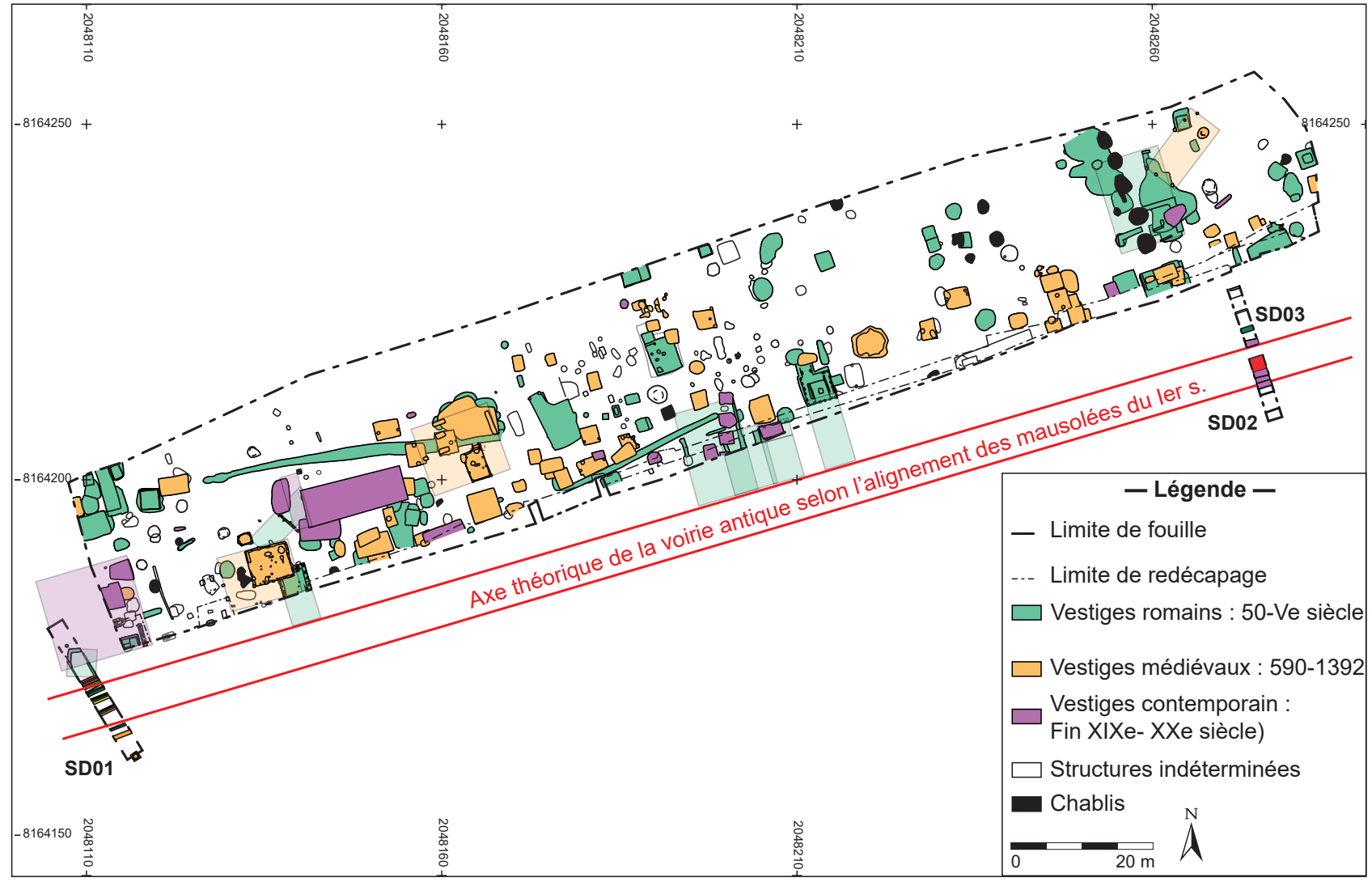

Fig. 1 : Occupations successives mises au jour au 2, route des Romains DAO : S. Guillotin.

[3] FLOTTE 2020.

[5] SCHNitZleR \& FLotTe 2017, p. 94-99.

[4] SChNitZler \& FLotTe 2017, p. 175-178. 
Enfin, entre mars et avril 2019, une dernière opération de fouille a été menée par Mathias Higelin (Archéologie Alsace) au niveau du nœud routier A35/ A351 et de la rue de Koenigshoffen. Elle a livré un espace funéraire daté de la fin du $\mathrm{I}^{\mathrm{er}}$ siècle et du $\mathrm{II}^{\mathrm{e}}$ siècle de notre ère constitué de sépultures secondaires à crémation en fosses et de tombes-bûchers, quelques caves antiques, un fossé d'époque médiévale et des aménagements contemporains.

\section{UNE SUCCESSION D'OCCUPATIONS INATTENDUE ET INÉDITE}

Les vestiges mis au jour au 2, route des Romains font état d'une occupation de longue durée comprise entre le I ${ }^{\text {er }}$ siècle de notre ère et 1392 , date de destruction de l'agglomération médiévale. Des traces d'activités et d'habitats remontant à la fin du XIXe siècle ou au début du XXe siècle ont également été reconnues (fig. $\mathbf{1}$ ). Si la découverte de la continuité de la nécropole et de l'habitat du Haut-Empire était attendue, il n'en est pas de même pour les autres éléments mis au jour. Ainsi, la réoccupation de l'habitat des II $^{\mathrm{e}}$ et III ${ }^{\mathrm{e}}$ siècles par une nouvelle occupation attribuable à l'Antiquité tardive et la mise au jour d'un habitat médiéval implanté par-dessus ces derniers restes ont constitué une surprise pour les archéologues; de même, la mise au jour de bâtiments et de fosses établies dans le secteur non constructible des fortifications de la ville après la guerre de 1870.

La particularité de ce site réside également dans le fait que cette succession de nouveaux habitats n'était pas «prévue ». Koenigshoffen n'avait en effet, jusqu'à ce jour, livré que des zones funéraires datées de la fin de l'occupation romaine à l'est et au nord du faubourg ainsi que quelques fonds de cabanes mérovingiennes sous I'actuelle église Saint-Paul ; aucune zone d'habitat médiévale densément organisée n'avait encore été découverte, alors même que les textes mentionnaient l'existence d'un hameau dispersé aux abords de la sortie ouest de la Strasbourg, le long de l'ancienne voie antique. Ce constat est le même concernant les quelques restes de bâtiments en terre et bois pourtant très récents découverts à I'ouest des parcelles fouillées et qui ne figuraient sur aucun plan des archives strasbourgeoises.

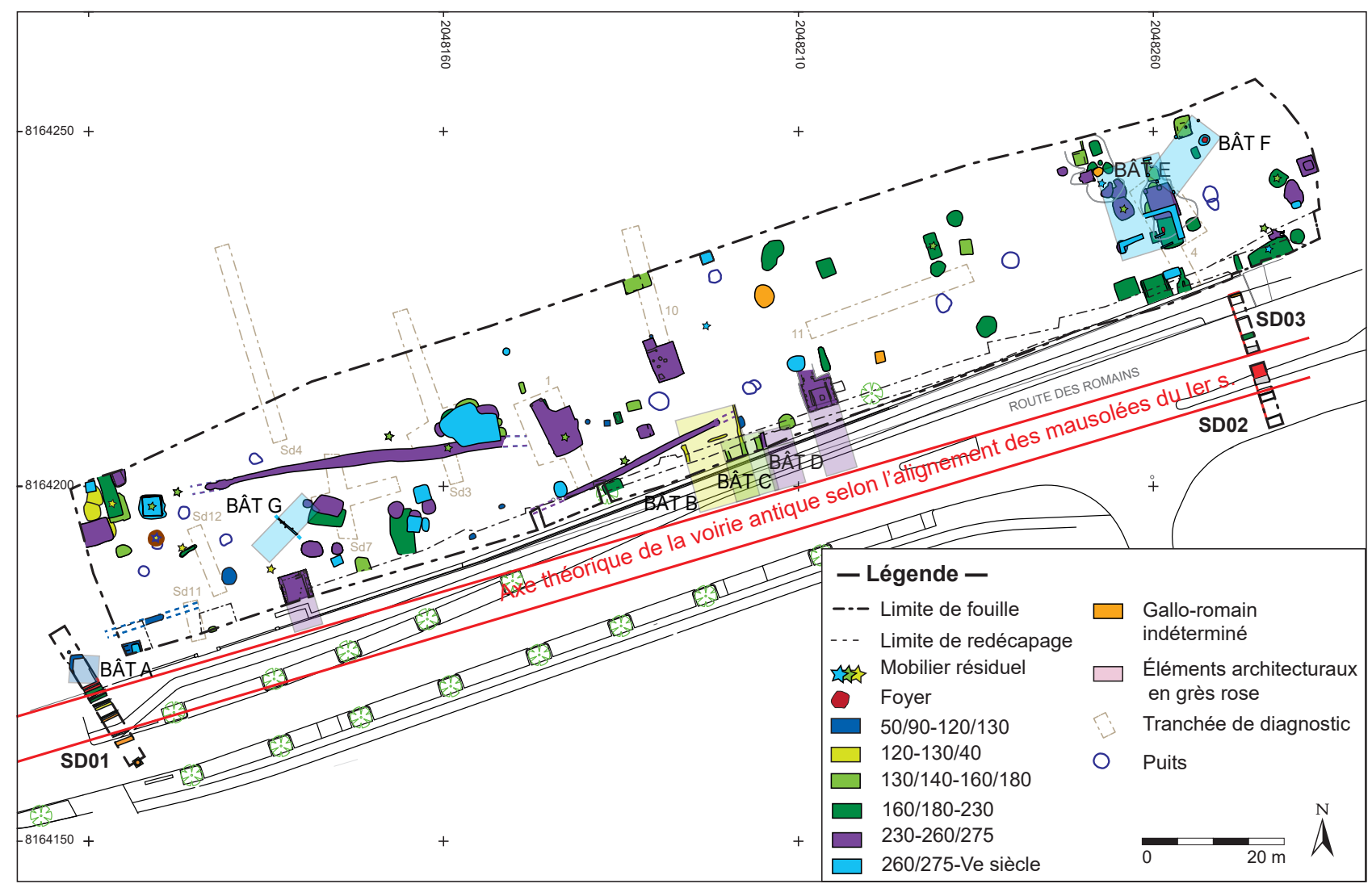

Fig. 2 : Plan des vestiges antiques mise au jour au 2, route des Romains DAO : S. Guillotin. 


\section{LES VESTIGES ANTIQUES (FIG. 2)}

Au 2, route des Romains, la période antique est marquée par trois occupations chronologiquement distinctes présentant chacune des caractéristiques différentes. Celles-ci s'échelonnent entre la première moitié du Ier siècle et le Ve siècle. Il est par ailleurs possible qu'à l'instar du site voisin du 8-20 route des Romains, une fréquentation plus ancienne du site ait existé comme en témoigne, entre autres, la découverte d'un fragment de bracelet laténien en pâte de verre.

Ainsi, la première occupation est à mettre en lien avec la nécropole du $\mathrm{I}^{\mathrm{er}}$ siècle, la seconde est attribuable à I'habitat qui vient se développer sur le site funéraire entre le second tiers du II ${ }^{e}$ siècle et le second tiers du III siècle, suite à l'arrivée de la VIII légion, tandis que la dernière, inédite, correspond aux restes d'un habitat du Bas- Empire établi sur les ruines de l'occupation précédente, détruite par un incendie.

\section{PROBLÉMATIQUE DE LA VOIRIE ANTIQUE}

Quelques fouilles anciennes avaient permis d'observer de façon ponctuelle la voirie antique sous le tracé de l'actuelle route des Romains [6]. La réalisation de deux tranchées de suivi de travaux sous cet axe contemporain, durant la fouille, devait permettre de documenter la voie antique et ses éventuels aménagements de bordure. Malheureusement, cet exercice n'a pas permis d'en trouver trace, alors même que les restes d'un enclos ou d'un mausolée funéraire ont été découverts dans le sondage 1 situé à l'ouest de la fouille, attestant du fait que le tracé de l'ancien axe ne devait pas être très éloigné de ce monument.

Deux hypothèses s'offrent à nous pour expliquer cette lacune : la première est que les nombreuses perturbations contemporaines en lien avec la voirie actuelle (fibre, éclairage, assainissement...) et les aménagements antiques et médiévaux de type cave enregistrés dans ces sondages ont oblitéré le tracé de la voie (fig. 2) ; la seconde est que ce tracé était peut-être légèrement décalé vers le sud en cet endroit du vicus de façon à laisser un espace entre les mausolées et l'espace viaire, puis plus tard, entre l'habitat qui succède à la nécropole et le decumanus principal.

\section{NOUVEAUX APPORTS SUR LA NÉCROPOLE MILITAIRE DE KOENIGSHOFFEN}

Les quelques vestiges mis au jour relatifs à la nécropole des légionnaires sont répartis à l'ouest de la fouille (fig. 3) : on recense une série de vestiges à caractère funéraire et des restes de parcellaire. Quantitativement, ces éléments sont beaucoup moins nombreux que sur le site du 8-20 route des Romains [7]. En effet, ce dernier a livré 18 ensembles funéraires alignés de manière régulière le long de l'antique voie décumane, alignement aujourd'hui connu sous le vocable $d^{\prime}$ « Allée des tombeaux ». Trois nouvelles inscriptions ont également été retrouvées, dont deux sur des stèles funéraires, confirmant le recrutement exclusif d'hommes originaires d'Italie, de Gaule Narbonnaise ou d'Espagne, qui étaient pour l'essentiel des soldats ou des vétérans de la II légion, laquelle a stationné à Strasbourg entre 15 et 43 apr. J.-C. Quelques monuments plus tardifs datant de la seconde moitié du Ier siècle ont également été dégagés. Enfin, quelques sépultures à crémation secondaires en fosses attribuables à des hommes de la VIII légion ont été signalées.

Sur notre site, si certains monuments funéraires, déjà arasés et démantelés à l'époque antique, lors de la fermeture de ce secteur de la nécropole, ont pu être définitivement oblitérés par les terrassements importants de la fin des années 1960, le maillage des aménagements funéraires liés à la nécropole du I ${ }^{\text {er }}$ siècle semble néanmoins beaucoup moins dense que celui observé au 8 , route des Romains. Ceci signifie que les parcelles fouillées étaient probablement situées à I'origine à la limite de la première nécropole militaire.

\section{DES AMÉNAGEMENTS À CARACTĖRE FUNÉRAIRE DE NATURES DIVERSES}

Le mobilier associé aux structures funéraires du 2, route des Romains permet d'établir que la portion de nécropole exhumée a été fréquentée tant par les hommes de la II ${ }^{e}$ que de la VIII légion. Parmi les vestiges encore préservés, on distingue un certain nombre de structures marquant plusieurs étapes de la cérémonie funéraire, en passant par la crémation à proprement parler, l'inhumation, le banquet funéraire, voire encore la commémoration des défunts. Malgré cette diversité, il convient de souligner une fois de plus le caractère lacunaire de ces vestiges et de mentionner que ce secteur funéraire devait à l'origine être plus riche que ce qui nous a été donné de découvrir à I'issue de cette opération préventive.

Les seuls restes attribuables à un monument ont été découverts sous le trottoir nord de la route des Romains (fig. 3, Bât. A). Il s'agit de deux tranchées formant un angle pouvant correspondre soit à des tranchées de récupération liées à un bâtiment, soit aux restes d'un enclos. Dans les deux cas, ce monument devait

[6] WATON \& FLOTTE 2017, p. 89.

[7] FLotTe 2020. 


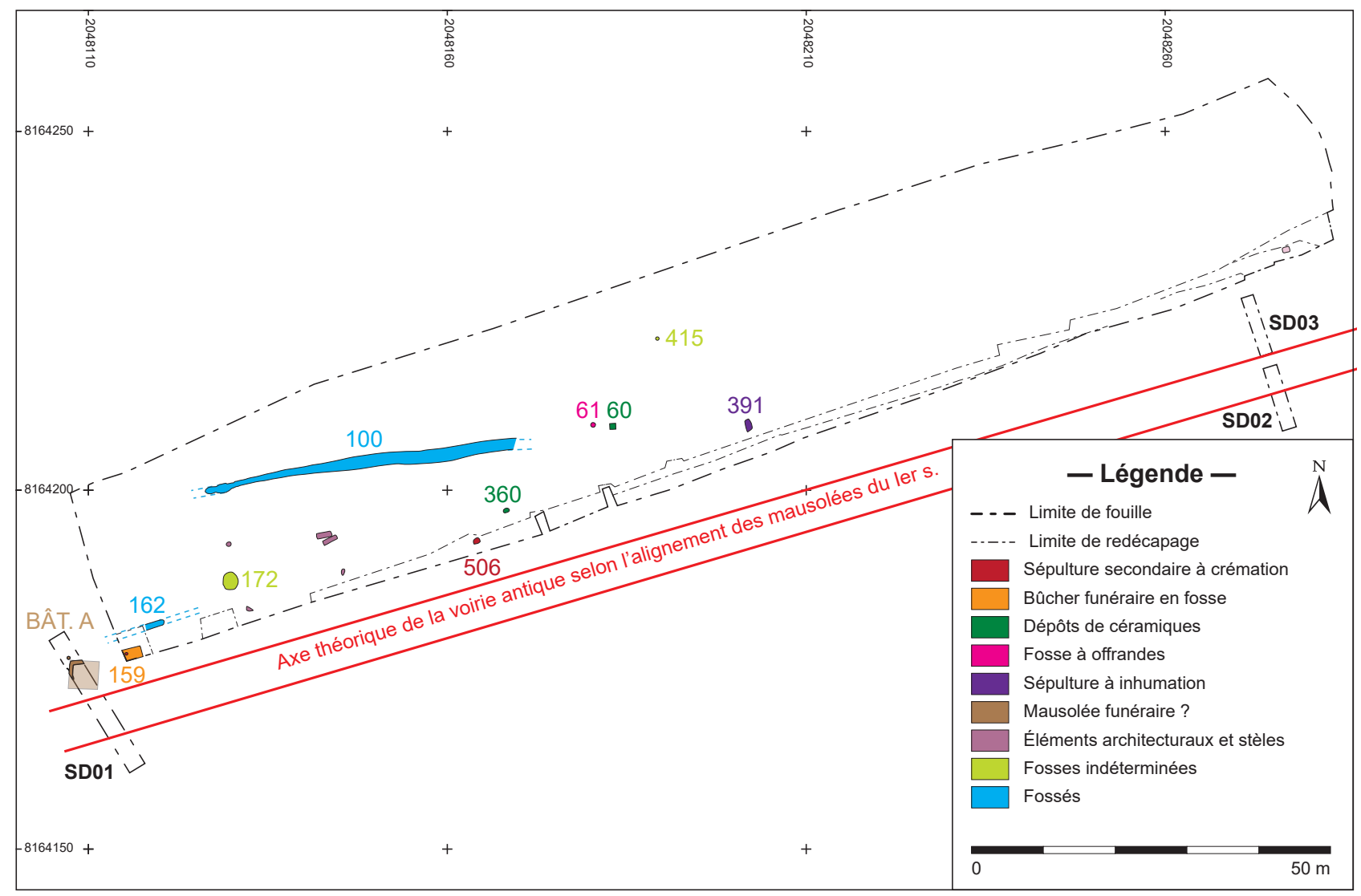

Fig. 3 : Carte de répartition des vestiges liés à la nécropole du Ier siècle DAO : S. Guillotin.

abriter une, voire plusieurs sépultures à crémation. La localisation, en dehors des limites de la plaine sportive, a préservé ce monument des terrassements ayant possiblement oblitéré des édifices similaires qui auraient pu se développer à hauteur de la limite sud de la fouille. L'angle sud-ouest de la fouille abritait les restes d'un bûcher funéraire en fosse n'ayant malheureusement pas pu être observé intégralement du fait de sa localisation (fig. 3, ST 159). Sa morphologie est identique à celle des bûchers aux parois inclinées et rubéfiées découverts en 2019 par Mathias Higelin sur les parcelles situées dans le prolongement du 2, route des Romains, la différence résidant uniquement dans le fait que cette structure à incinération n'a pas été transformée en tombe après la combustion du corps, à I'instar des bûchers mis au jour par Archéologie-Alsace. Cependant, le fond de cet aménagement a fait I'objet d'un recreusement après la vidange du bûcher, afin d'y déposer un lot de balsamaires en verre fondus par I'action du feu. Le type de ces contenants a permis de définir que le bûcher a servi à l'époque où la légion II stationnait encore à Argentorate.

Non loin du bûcher se trouvait une fosse qui présentait un comblement cendreux et des tessons brûlés contemporains du dépôt de balsamaires (fig. 3, ST 172).
Celle-ci pourrait correspondre à une fosse de curage de bûcher et signaler la présence d'une autre structure liée à la combustion des corps dans le secteur.

L'unique sépulture à crémation qui nous est parvenue présente les caractéristiques des tombes les plus simples attribuables à la VIII légion que l'on rencontre à Koenigshoffen, la datation de la céramique constituant ce dépôt ayant par ailleurs confirmé cette assertion. Elle se présentait sous la forme d'une fosse aménagée dans le lœss, sur le fond de laquelle était déposé un pot de stockage en céramique qui recelait les restes osseux et une moitié de cruche déposée à l'envers, goulot enterré dans le sol, geste censé représenter I'acte de libation (fig. 3, ST 506 et fig. 4). La fosse était endommagée par les racines de la haie de thuya qui délimitait le terrain sportif. En raison de son état de conservation, il nous a été impossible de déterminer si cette tombe était intégrée ou non à un mausolée, à un simple enclos, ou bien si elle pouvait être simplement marquée en surface d'une stèle. À proximité immédiate de la sépulture se trouvait un dépôt de céramique qui comportait trois moitiés supérieures de cruches (fig. 3, ST 361), également choisies pour symboliser l'action de libation. Ce voisinage pourrait indiquer que ce dépôt a été installé suite à l'inhumation des restes dans la fosse. 


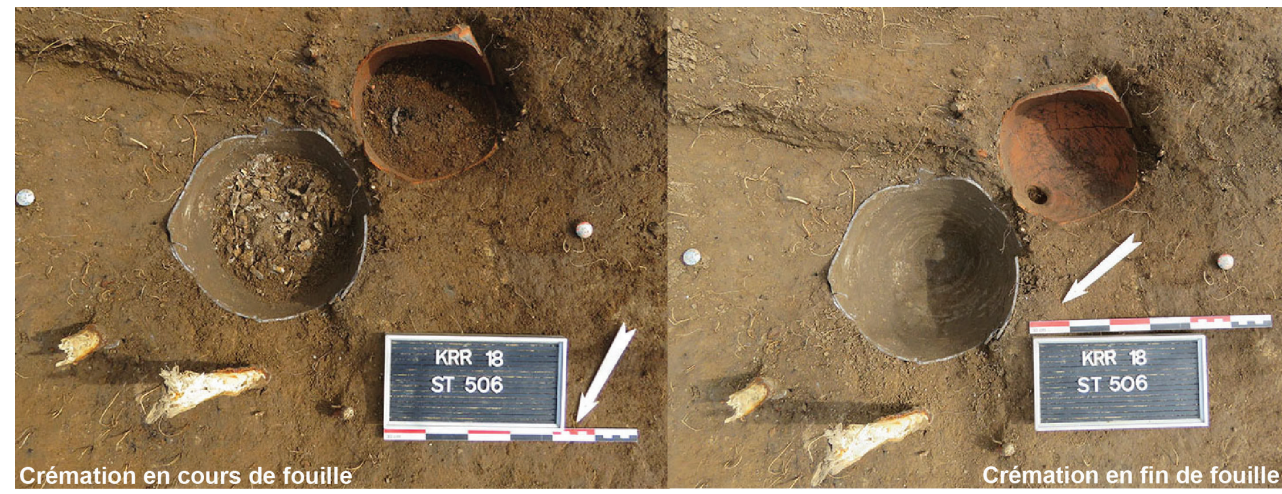

Fig. 4 : différentes étapes de la fouille de la sépulture à crémation 506. Photo:

ANTEA-Archéologie.

Une inhumation d'enfant dépourvue de mobilier d'accompagnement figurait également parmi les restes funéraires (fig. 3, ST 391). Sa localisation en bordure de la route des Romains pourrait suggérer son intégration à la nécropole militaire, mais sa datation par ${ }^{14} \mathrm{C}$ (1925 \pm 30 BP, soit de 4 à 134 apr. J.-C. - datation à $2 \sigma)$ permet également de supposer que l'enfant a été inhumé avant l'installation de cet ensemble. La présence de sépultures d'enfants en contexte militaire n'est pas inconnue mais reste rare. À ce titre, on mentionnera que l'un des tombeaux mis au jour au 8-20 route des Romains, remployé à la fin du I Ier siècle, a livré les restes d'une crémation d'immature [8].

Outre ces restes funéraires, on dénombre une fosse à dépôt d'offrandes (fig. 3, ST 60 et fig. 5), jouxtant un dépôt de céramique comportant des restes de récipients brisés sous l'action du feu (fig. 3, ST 61), ces deux aménagements ayant très certainement été mis en place simultanément, au vu de la similitude des récipients qui les composaient. Ces restes devaient à I'origine être associés à une crémation disparue suite à l'arasement mécanique du terrain. La fosse à dépôt d'offrandes recelait un assemblage de récipients miniatures en sigillée, des pots de stockage miniatures et des restes de cruches, des résidus de flacons en verre et quelques ossements de faune. La plupart des éléments étaient brisés sous l'action du feu et réunis en un amas qui présentait une forme circulaire suggérant un enfermement dans un contenant souple. L'assemblage ainsi constitué est caractéristique des fosses rituelles réalisées suite à la crémation du corps dans le cadre du banquet funéraire. La présence de récipients miniatures, qui pouvaient contenir des offrandes, évoque en effet plus la pratique d'un simulacre de banquet qu'un réel banquet destiné à commémorer la mémoire du défunt. Le fait que la plupart des récipients ont été brisés par le feu indique en effet que les éléments étaient déposés sur le bûcher funéraire. Dans ce contexte, il est intéressant de préciser que les récipients étaient incomplets, le plus souvent représentés par un seul fragment permettant de déterminer l'objet, ce qui montre ici que ces fragments ont été sélectionnés parmi les restes du bûcher. Ce geste est à rapprocher du rite de la pars pro toto pratiqué lors des cérémonies funéraires romaines, qui consiste à choisir la part symbolique d'un objet destiné à représenter son intégralité. Enfin, la présence de restes de cruches fait également référence à la pratique de la libation.

\section{DES RESTES DE LAPIDAIRES}

Outre ces aménagements funéraires variés laissant entrevoir l'existence d'un noyau funéraire plus complexe, la mise au jour de quelques éléments architecturaux plaide en faveur de la présence de monuments funéraires qui devaient être érigés en bordure de la voie antique. Ainsi, outre les deux stèles funéraires dédiées à deux légionnaires de la légion VIII, avec leurs inscriptions inédites (qui sont présentées à la suite de cet article, dans ce même numéro d'Archimède. Archéologie et histoire ancienne [9]), des éléments lapidaires en grès et en calcaire ont été retrouvés en position secondaire dans des fosses et un puits, dans un périmètre restreint à l'ouest de la fouille et en bordure de la berme sud. La plupart de ces blocs s'apparentent à des chaperons en grès destinés à couvrir les murs des mausolées funéraires, similaires à ceux découverts sur le site du 8-20 route des Romains [10]. Si la découverte de ces monolithes indique bien que cette portion de la nécropole militaire a été démantelée au début du $\mathrm{II}^{\mathrm{e}}$ siècle en raison de la pression foncière et du développement du vicus, il nous est impossible de déterminer précisément d'où provenaient les restes architecturaux ni s'ils ont fait l'objet de déplacements successifs. La surprise constituée par la trouvaille des deux stèles funéraires au 
Fig. 5 : différentes étapes de la fouille de la fosse à dépôt d'offrandes 60.

Photo :

ANTEA-Archéologie.

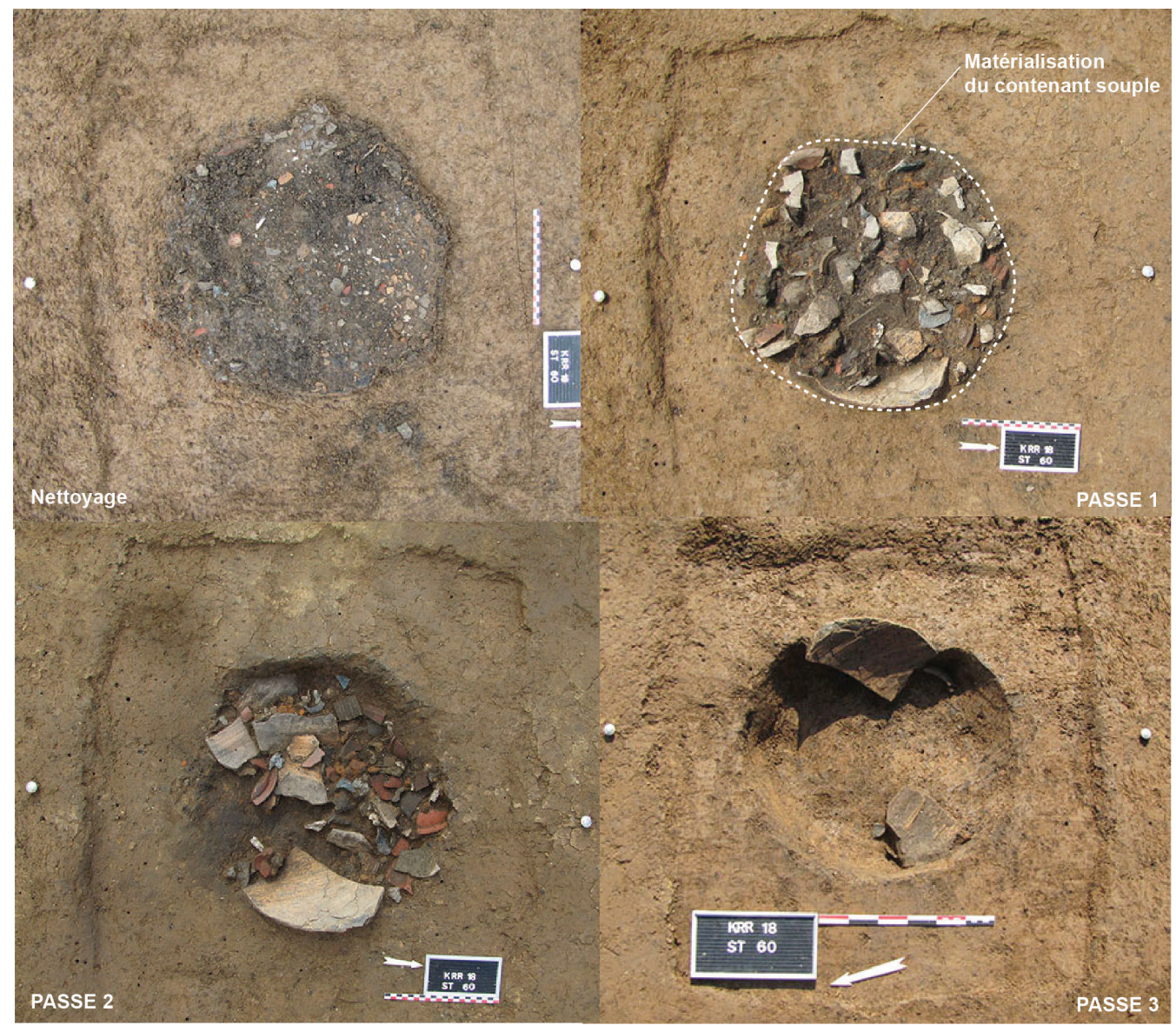

fond d'une fosse datée de la fin du XIX`e ou du tout début du XXe siècle illustre bien que ces stèles ont été enfouies au moins une première fois suite à la destruction de la nécropole, puis, découvertes et ré-enfouies à l'époque contemporaine. Cependant, étant donné le volume et la taille de certains blocs, notamment des stèles, il certain qu'ils n'ont pas été déplacés sur une longue distance et que leur emplacement d'origine se situait non loin de leur lieu de découverte.

\section{DES DÉLIMITATIONS PARCELLAIRES LACUNAIRES}

Les éléments de parcellaire liés à la nécropole légionnaire correspondent à deux tronçons de fossés incomplets (fig. 6, ST 100 et 162), dont I'un a livré les restes d'une jument datés par ${ }^{14} \mathrm{C}$ de $1925 \pm 30$ $\mathrm{BP}$, soit de 4 à 134 apr. J.-C. (datation à 20) associés à un dupondius de Vespasien (en circulation entre 69 et 79) et le second de la céramique datée du milieu du $\mathrm{I}^{\mathrm{er}}$ siècle. Le caractère fortement lacunaire de ces deux tronçons, perturbés par l'aménagement de fonds de cabanes médiévaux puis par les terrassements mécaniques préalables à l'aménagement de la plaine sportive, ne nous permet pas de savoir ce que délimitaient précisément ces fossés. Malgré tout, il est possible d'émettre des hypothèses quant à la chronologie de leur utilisation en croisant plusieurs données.
Ainsi, le premier ouvrage en fonction, dont seule une portion réduite a pu être observée, pourrait être le fossé 162 , en raison de son orientation identique à celle des mausolées mis au jour au 8-20 route des Romains. Cet ouvrage pourrait par conséquent avoir été utilisé durant la période de fonctionnement de la nécropole de la II légion, ce que suggère également la datation du matériel livré par son comblement. Le fossé 100 , qui a livré une dépouille de jument et une monnaie, dispose quant à lui d'une orientation identique à la fosse à dépôts d'offrandes et au reste de monument découvert sous le trottoir nord de la route des Romains (fig. 6, ST 60 et Bâtiment A). Ces données ainsi que la datation du matériel archéologique découvert dans le comblement de ces différents éléments plaident en faveur de leur incorporation à un noyau funéraire plus récent dédié aux soldats et vétérans de la VIII légion. La présence du dépôt de jument est intéressante, car elle suggère un acte en lien avec la protection des défunts. La jument est en effet l'animal emblème de la déesse-mère Epona dont l'une des responsabilités consiste à assurer le voyage des défunts vers l'au-delà ; de la même façon, le cheval occupe une place importante dans le monde militaire antique et figure souvent sur les stèles funéraires de légionnaires ou de soldats auxiliaires. La présence de cette inhumation au sein du fossé pourrait par conséquent trouver une réponse dans le rôle psychopompe que joue cet animal en Gaule romaine. 
QUELQUES PISTES SUR LE DÉVELOPPEMENT DE LA NÉCROPOLE MILITAIRE

Si nombre de structures nous font cruellement défaut pour assurer une bonne compréhension de cette nouvelle portion de la première nécropole militaire, nous avons tout de même pu tirer quelques indices sur la façon dont elle pouvait s'agencer. A priori, on constate deux orientations différentes dans la structuration des aménagements : la première orientation est conforme à celle de « l'Allée aux tombeaux » du 8-20 route des Romains et se développe très certainement parallèlement au tracé du decumanus principal. Elle concerne le fossé se développant le plus en bordure de la berme sud (ST 162), le bûcher funéraire (ST 159) et la sépulture à crémation (ST 506). La seconde regroupe le fossé avec le dépôt de jument (ST 100), l'édicule funéraire (Bâtiment A) et la fosse à dépôts d'offrandes (ST 60). Le mobilier archéologique associé à ces vestiges confirme que la première orientation était liée à la IIe légion tandis que la seconde l'était à la VIII légion. Cette situation permet d'envisager l'existence d'un premier noyau se développant aux abords des $24-28$ et 8-20 route des Romains, exploité par les militaires de la légion II, d'une timide réoccupation de ce secteur par les premiers défunts de la VIIIe légion, puis du développement d'un second noyau funéraire par l'occupation de terrains situés plus à l'est, le long de la voie décumane, c'est-àdire à hauteur du 2, route des Romains, entre la fin du I $\mathrm{I}^{\text {er }}$ siècle et le démantèlement de la nécropole vers le début du second tiers du II ${ }^{e}$ siècle. Cette hypothèse semble confirmée par les découvertes effectuées par Mathias Higelin en 2019, dans le prolongement des parcelles fouillées, qui montrent qu'après le démantèlement des tombeaux des 8-20 et 2, route des Romains au profit de l'extension de l'habitat, la nécropole militaire s'est développée vers l'est, en direction du camp. Pour finir, on signalera que la faible densité d'occurrences funéraires mises au jour au 2, route des Romains ne peut pas simplement être expliquée par la disparition des vestiges à la fin des années 1960, mais par le fait que I'on se situait très probablement en limite de ces premiers noyaux de la nécropole militaire. L'analyse de la répartition des vestiges funéraires repérés durant cette opération a en effet montré que les restes funéraires étaient uniquement localisés aux abords de la bordure de la moitié ouest de la fouille, l'inhumation d'un enfant étant a priori le dernier élément funéraire mis au jour. Phénomène intéressant, le premier habitat qui se met en place après le démantèlement de la nécropole ne se développera pas non plus au-delà de cette zone.

[11] Baudoux \& CicutTA 2017, p. 106.
L'HABITAT ET LE QUARTIER ARTISANAL DU HAUT-EMPIRE (FIG. 6)

Nos connaissances sur les premiers habitats du faubourg sont encore extrêmement limitées. On citera notamment la découverte de restes ténus d'une occupation d'époque augustéenne, de nature indéterminée, à l'extrémité ouest de Koenigshoffen. Les premiers vestiges d'habitat clairement identifiés ont été mis au jour dans la partie centrale de l'agglomération actuelle, au 100, route des Romains, et regroupent des bâtiments à pans de bois associés à un atelier de potiers daté par archéomagnétisme entre 40 et 70 [11]. L'agglomération prend son essor à la fin du I ${ }^{\text {er }}$ siècle, suite à l'arrivée de la VIII légion. On observe ainsi le développement de nouveaux habitats à vocation artisanale et la mise en place d'autres ateliers de potiers situés dans le secteur de la rue du Schnokeloch et au 42 rue des Capucins, puis dès le début du II ${ }^{e}$ siècle, le démantèlement de la première nécropole militaire au profit de nouveaux habitats et quartiers artisanaux se développant en direction du camp. C'est à ce dernier mouvement d'urbanisation qu'appartiennent les restes d'habitats découverts au 8-20 route des Romains, entre 2014 et 2015 , et sous I'ancienne plaine sportive de Koenigshoffen, en 2018.

\section{L'HABITAT ET SES ARRIÈRE-COURS}

Au 2, route des Romains, les premières parcelles d'habitat sont aménagées à partir du second tiers du II siècle, après arasement de l'espace funéraire. Comme mentionné précédemment, une grande partie des éléments structurants constituant cet habitat a été oblitérée ou amoindrie par les terrassements préalables à l'aménagement du terrain de I'ASPTT, ce qui fait que de nombreux plans de bâtiments, fossés ou palissades permettant de définir l'existence de parcelles ont disparu, offrant aux archéologues une vision incomplète de l'agencement originel des aménagements.

La nature des vestiges en creux exhumés (latrines, puits, fosses d'extraction, silos...) a cependant permis d'établir que la fouille a touché l'arrière des habitats qui se développaient en bordure du decumanus principal. Seules quelques substructures très arasées localisées à l'ouest et en zone médiane de la fouille ont permis de définir la présence de bâtiments fondés sur vide sanitaire, les caves aménagées à l'arrière de ces bâtiments constituant le plus souvent les seuls témoins de ces édifices.

Les nombreux remaniements observés sur le seul bâtiment détecté en bordure de la route des Romains, ainsi que sur plusieurs des caves, attestent que la trame de I'habitat du Haut-Empire a perduré sans évolution majeure jusqu'au début du dernier tiers du III ${ }^{\mathrm{e}}$ siècle. 


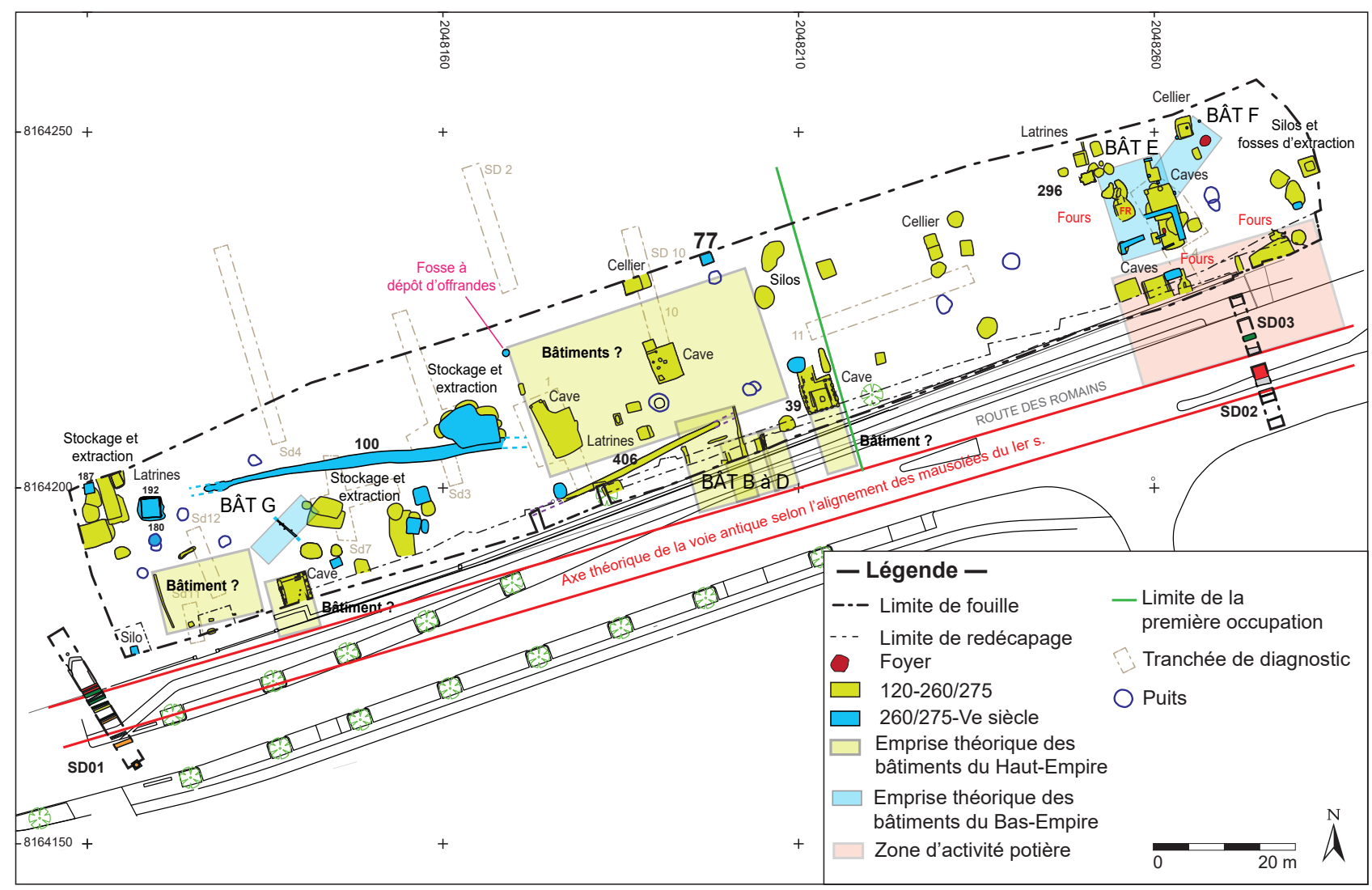

Fig. 6 : Occupations du Haut et du Bas-Empire. DAO : S. Guillotin.

Dans un premier temps, I'occupation reste cantonnée à l'ouest des parcelles fouillées, sa limite étant marquée par la cave 39 (fig. 6). Vers le milieu du II siècle, I'occupation s'étend sur les terrains situés plus à l'est où I'on retrouve essentiellement de petits celliers et des silos, indiquant que cet espace était plutôt dévolu à des activités agricoles.

Entre la fin du II ${ }^{\text {e }}$ siècle et le début du IIIe siècle, on note à l'ouest l'apparition d'un fossé (Fig. 6, ST 406), à I'arrière du bâti situé en bordure de la route des Romains et deux nouvelles caves environnées de fosses de fondation arasées appartenant à des bâtiments de plain-pied. Ces témoins signalent la création probable de deux nouvelles unités d'habitat tournées vers la voie principale et séparées des occupations de bord de voie par ce nouvel élément de parcellaire.

\section{UNE ACTIVITÉ POTIĖRE IMPORTANTE...}

Dans un intervalle de temps identique à la mise en place de ces nouvelles unités, le secteur agricole situé à l'est de la fouille est restructuré au profit d'installations dédiées à l'artisanat potier qui perdureront un demi-siècle. Ces unités sont équipées de six fours, environnés de pièces-semi excavées faisant office de locaux artisanaux et de latrines (fig. 6). Le répertoire des formes réalisées dans ces fours était classique, caractéristique des productions régionales que I'on retrouve dans les officines en fonction entre le milieu du $\mathrm{II}^{\mathrm{e}}$ siècle et le dernier tiers du $\mathrm{III}^{\mathrm{e}}$ siècle et était exclusivement destiné à alimenter les quartiers civils situés en périphérie du camp ; à titre d'exemple, on peut citer la découverte de formes de récipients encore inédites dans les assemblages du III ${ }^{\mathrm{e}}$ siècle de la partie de canabae mise au jour à I'Hôpital Civil en 2012, pour lesquelles nous avons eu la surprise de découvrir qu'elles étaient produites dans les fours du 2, route des Romains [12].

Un pan important de la production concernait des récipients multifonctions tels que des pots, marmites et jattes, destinés à la cuisson et à la préparation des aliments, mais également des cruches aux profils variés, de petit ou grand module. L'atelier réalisait également de la vaisselle de table telle que des gobelets à boire ou des assiettes [13].

L'analyse des fours, de la production et du matériel exogène associé aux différents ensembles suggère l'existence d'un premier atelier situé en bordure de

[12] MURER 2016.

[13] GOHIER \& MURER 2020. 


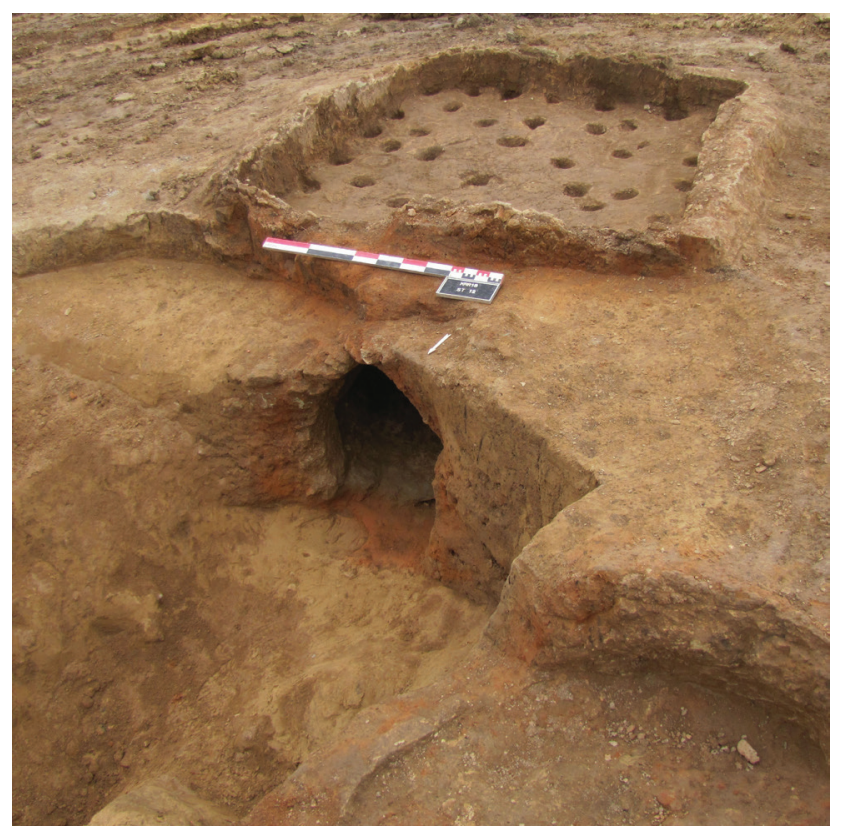

Fig. 7 : four de potier en cours de fouille. Photo : ANTEA-Archéologie.

la voie décumane, à hauteur de l'actuelle route des Romains, et qui se serait développé au fil du temps vers l'arrière des parcelles. Les indices stratigraphiques et structurels mis en évidence lors de l'étude des structures de production permettent d'établir que la portion d'atelier touchée par la fouille a dans un premier temps fonctionné avec des fours de module moyen, de plan circulaire ou quadrangulaire (fig. 7) et dotés d'une fosse de travail, pour évoluer vers une production moins importante, avec des fours de petite dimension, hors sol et dépourvus de fosses de travail.

La reprise des données post-fouille a permis de mettre en exergue l'existence au sein de ce secteur artisanal, et ce pour la première fois à Koenigshoffen, de rites liés à la clôture des fours de potiers. Ces gestes pratiqués par la confrérie des potiers lors de l'abandon d'une ou plusieurs structures de chauffe, étaient visibles sur le terrain de différentes manières : récipients ratés, complets ou semi-complets, déposés dans la chambre de cuisson des fours, sous la sole préalablement cassée à cet effet ou encore sur le fond ou le dernier niveau de comblement des fosses de travail au débouché des alandiers ; présence dans les fosses dépotoirs des fours de nombreux restes de cruches issues de la production, présentant des traces de mutilations volontaires telles qu'on les retrouve généralement en contexte funéraire ou rituel, mais également d'objets particuliers dont la fonction tournait autour du feu ou encore fabriqués à l'aide du feu (lampe à huile, lanterne, creuset, fer de lance...), symbole de l'activité potière. Tous ces éléments sont généralement associés aux dépôts que I'on retrouve dans les contextes de clôture de four où des gestes structurés ont été mis en évidence dans les ateliers du Nord de la Gaule [14].

Enfin, on peut associer à ces pratiques remarquables une structure tout à fait particulière : il s'agit de latrines intégrées au complexe artisanal (fig. 6, ST 296) qui recelaient les restes d'une trentaine de jeunes brebis et d'immatures de même sexe, auxquelles était mêlée de la vaisselle particulière (récipients miniatures en sigillée, mortiers en sigillée, gobelets à boire...) présentant des traces de bris volontaire et de découpe, un coquillage de type triton et une monnaie en argent (fig. 8). Cet assemblage atypique met en évidence les reliquats d'un banquet important ayant nécessité l'abattage d'un grand nombre de bêtes en âge de se reproduire et de futures brebis. Les restes d'un banquet au sein de l'atelier peuvent également être mis en lien avec les pratiques de dépôts observées dans les fours et leurs structures de rejets et être assimilés à une commémoration liée à l'abandon de I'atelier.

\section{...ET AUTRES INDICES D'ARTISANAT}

Comme cela a été précisé en introduction, les hasards de la prescription ont fait que la fenêtre de fouille a principalement touché l'arrière des habitats se développant en bordure de la voie principale traversant le vicus. De cela résulte l'importante présence sur le site de latrines, puits, silos ou fosses d'extraction, structures d'équipement et de stockage généralement intégrées aux arrière-cours et jardins des habitats en bandes. Le nombre important de ces vestiges s'explique également par le fait que suite à leur fermeture, ces structures sont remplacées par d'autres, souvent aménagées à côté puis utilisées comme fosses détritiques. Le matériel conséquent livré par le comblement de ces dépotoirs (rejets de cuisine, récipients brisés...) a de cette façon constitué une source intéressante pour la connaissance des pratiques alimentaires ou artisanales du secteur fouillé. L'analyse des éléments a permis de définir qu'outre l'artisanat de la poterie, les occupants pratiquaient également la boucherie et la cornetterie (fig. 9).

Le travail du fer est attesté par la présence de résidus de parois de fours scorifiées, des scories de fond de foyer ou encore des réductions de scories. Aucun four n'a cependant été mis au jour sur le site mais ces foyers pourraient faire partie des substructures

[14] Willems \& Favennec 2019. 


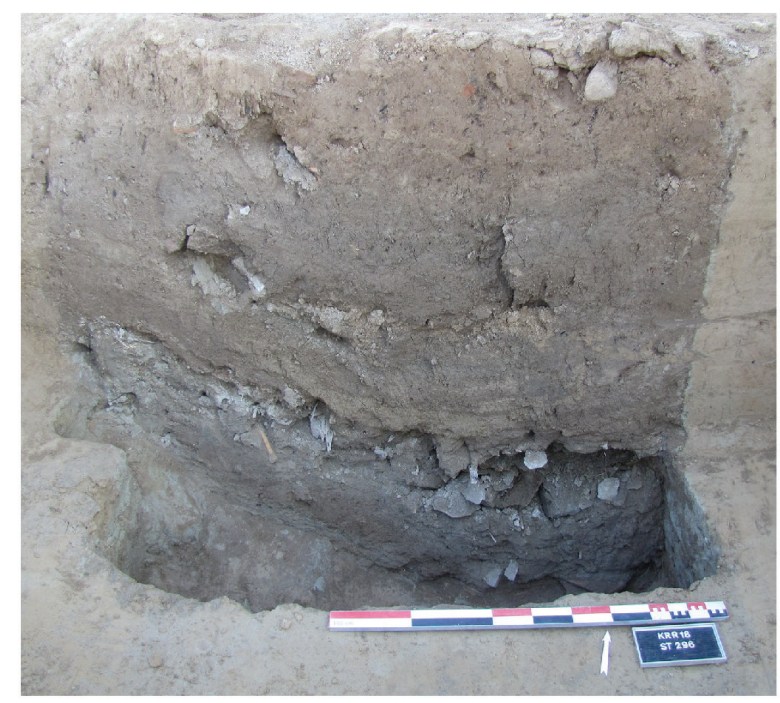

Coupe est-ouest dans les latrines

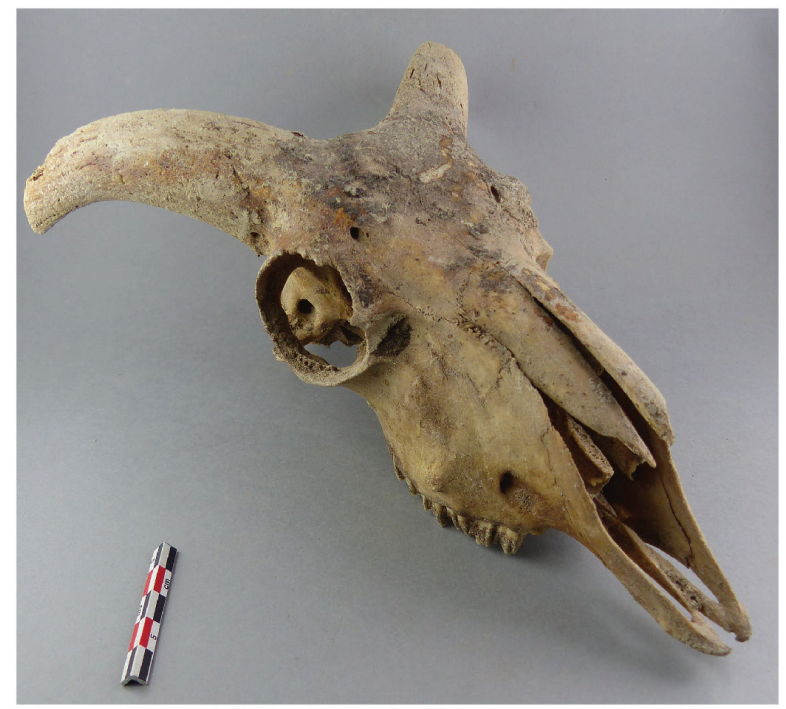

Crâne de brebis

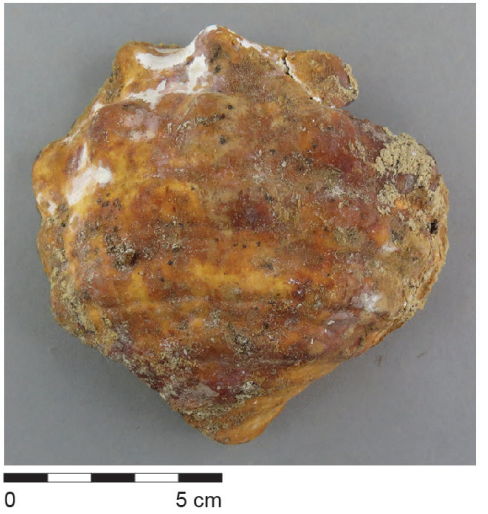

Triton géant
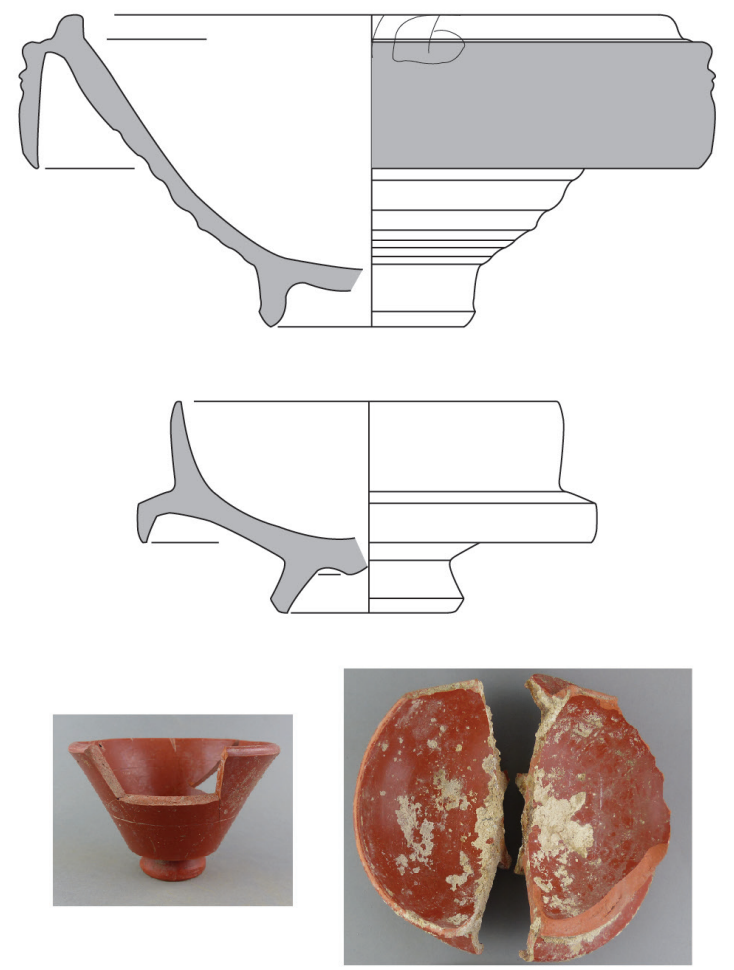

Trace de découpe et bri volontaire sur des récipients en sigillée de petit module
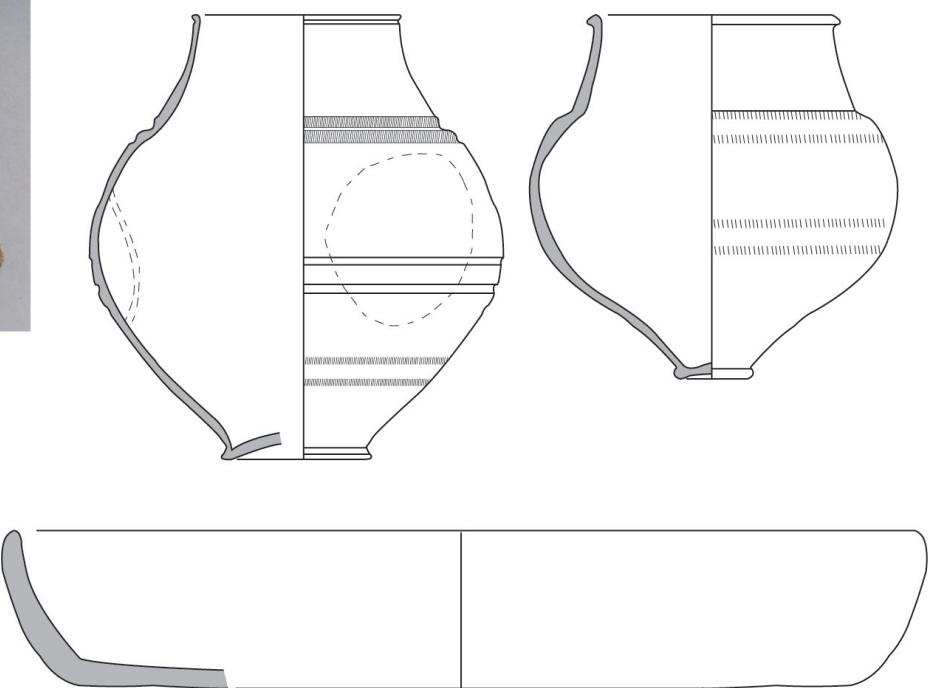

Exemple de matériel céramique découvert dans le comblement de la fosse d'aisance

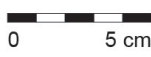

Fig. 8 : exemple d'assemblage de mobilier issu des latrines 296.

Photo : ANTEA-Archéologie. DAO : A. Murer. 


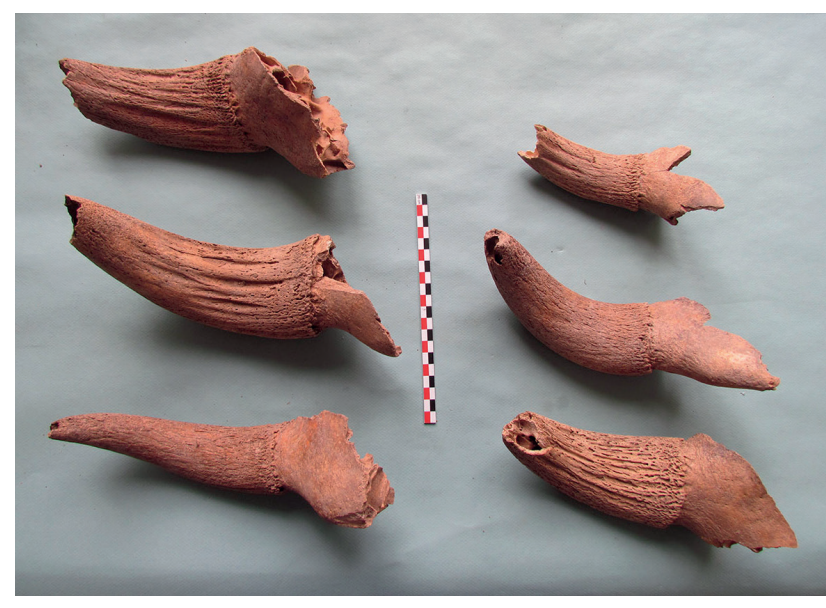

Fig. 9 : cornes de bovins découpées. Photo : M. Fabre.

disparues suite aux travaux préalables à l'aménagement du stade. Une plus forte densité de ce type de restes à l'ouest de la fouille pourrait indiquer que les opérations de réduction du minerai ont eu lieu dans ce secteur, où ont également été retrouvées une scorie, quelques coulures en alliage cuivreux et de plomb.

\section{UNE DESTRUCTION PAR LE FEU}

Les installations du Haut-Empire sont détruites par un important incendie entre le milieu et le début du dernier tiers du III siècle, dont les traces ont été observées dans le comblement et sur les parois de toutes les caves du site. La présence de sigillées estampillées dont la production ne dépasse pas 260 dans les fosses de rejet des fours, souligne que la fin de l'activité potière pourrait être liée à ce drame.

\section{UNE CONTINUITÉ DE L'OCCUPATION DURANT LE BAS-EMPIRE (FIG. 6)}

En Alsace, la période connue sous le terme de BasEmpire débute vers $260 / 270$; de manière générale elle constitue une transition entre l'Antiquité et le haut Moyen Âge. Elle se caractérise comme une période d'instabilité durant laquelle ont lieu les premiers raids germaniques au niveau du limes. Cette crise se traduit essentiellement par de profonds bouleversements économiques et sociaux, ainsi que par la rétractation des noyaux urbains.

La découverte de vestiges d'habitats datés du BasEmpire à Koenigshoffen était encore inédite à ce jour, seuls quelques zones funéraires avaient été mis au jour à la Porte Blanche [15] ou encore rue du Rail et rue du Chemin de fer [16].

Suite à la violente destruction de l'habitat, le site est réinvesti et reconstruit. La reprise systématique des anciens bâtiments ou unités d'habitat souligne que cette réoccupation s'est effectuée rapidement après la catastrophe ; cette supposition est également étayée par la datation du matériel céramique associé aux vestiges, dont la plus ancienne remonte au dernier tiers du III siècle. La découverte de tessons de céramique plus tardifs et d'un bol bas complet, de type franc, dans le comblement supérieur de latrines plaide en faveur d'une occupation jusqu'au Ve siècle.

\section{DES RESTES TÉNUS D'HABITAT ET DES INDICES D'UN NOYAU FUNÉRAIRE}

Seules trois structures d'habitat très arasées ont pu être rattachées à cette phase : deux bâtiments de plain-pied et un bâtiment hors sol (fig. 6, BÂT. E, F et $G$ ), mais I'on suppose que les anciens bâtiments ont pu être réoccupés, comme c'est souvent le cas durant cette période. Plusieurs structures d'équipement telles que des latrines et des silos ont pu être associées à ces bâtiments. L'une des latrines (fig. 6, ST 77) présentait sur son fond un dépôt singulier : un collier constitué de perles en pâte de verre, en or, en bois et en hématite, des restes de cochon immature, de canard et de chouette chevêche, le tout recouvert de gros fragments de panse d'amphore (fig. 10). La localisation de cette structure en bordure de la fouille et la méconnaissance de son contexte ne permettent pas de comprendre la signification de ce dépôt, comme nous avons pu le faire dans le cas des latrines intégrées à l'atelier de potiers. Cependant, il paraît évident que ce dépôt peut être rapproché des découvertes réalisées ces dernières années en contexte d'habitat dans la région de Strasbourg, qui constituent probablement des dépôts réalisés dans le cadre de la sphère familiale ou artisanale afin d'assurer la prospérité, la fertilité ou qui peuvent pour certains être interprétés comme des dépôts de fondation...[17].

La présence d'une fosse à dépôt d'offrandes située en limite nord de la fouille constitue le seul indice qu'un petit secteur funéraire devait y exister (fig. 6, ST 89). Les infrastructures qui devaient être associées à cette fosse se développaient soit par delà la berme du chantier, mais ont très certainement été oblitérées par les travaux de terrassements liés à la construction de la plaine sportive.

[15] SCHNITZLER 2017, p. 230.

[16] SCHNITZLER 2017, p. 258.

[17] On pense entre autres à la découverte d'un dépôt de tortue dans un coffre associé à de la céramique dans un silo du site de Brumath, 8, rue du Collège (Murer 2013), un dépôt associant deux chaussures et d'autres objets dans l'un des puits du site de l'Hôpital Civil de Strasbourg ou encore un dépôt de vase enterré à la base de l'alandier d'un des séchoir-fumoir du même site (Murer 2016). 
Cette fosse a livré un lot de coupes et gobelets à boire miniatures, un bord d'amphore, un fragment de bouteille en verre, les restes d'un fourreau d'épée, deux épingles et un jeton en os et des restes de faune.

\section{VERS UNE CONTINUITÉ DE L'OCCUPATION DU HAUT-EMPIRE}

Les fouilles menées ces dernières années au nord de la route des Romains ont montré que toutes les portions d'habitat ont été ruinées par des incendies après le milieu du III ${ }^{\text {e }}$ siècle. La question que I'on peut par conséquent se poser est de savoir s'il s'agit d'un seul et même incendie, et si celui-ci peut être lié à un raid germanique, bien qu'à notre connaissance, les sources ne mentionnent rien de tel. La découverte d'un noyau $d$ 'habitat au-dessus des vestiges ruinés au 2 , route des Romains permet toutefois d'établir que le vicus a connu une crise, quelle que soit son origine, et témoigne d'une rétractation du tissu urbain puisque les autres habitats du secteur n'ont jamais été reconstruits.

\section{Perles et pendeloques composant le collier de la fosse d'aisance $\mathbf{7 7}$}
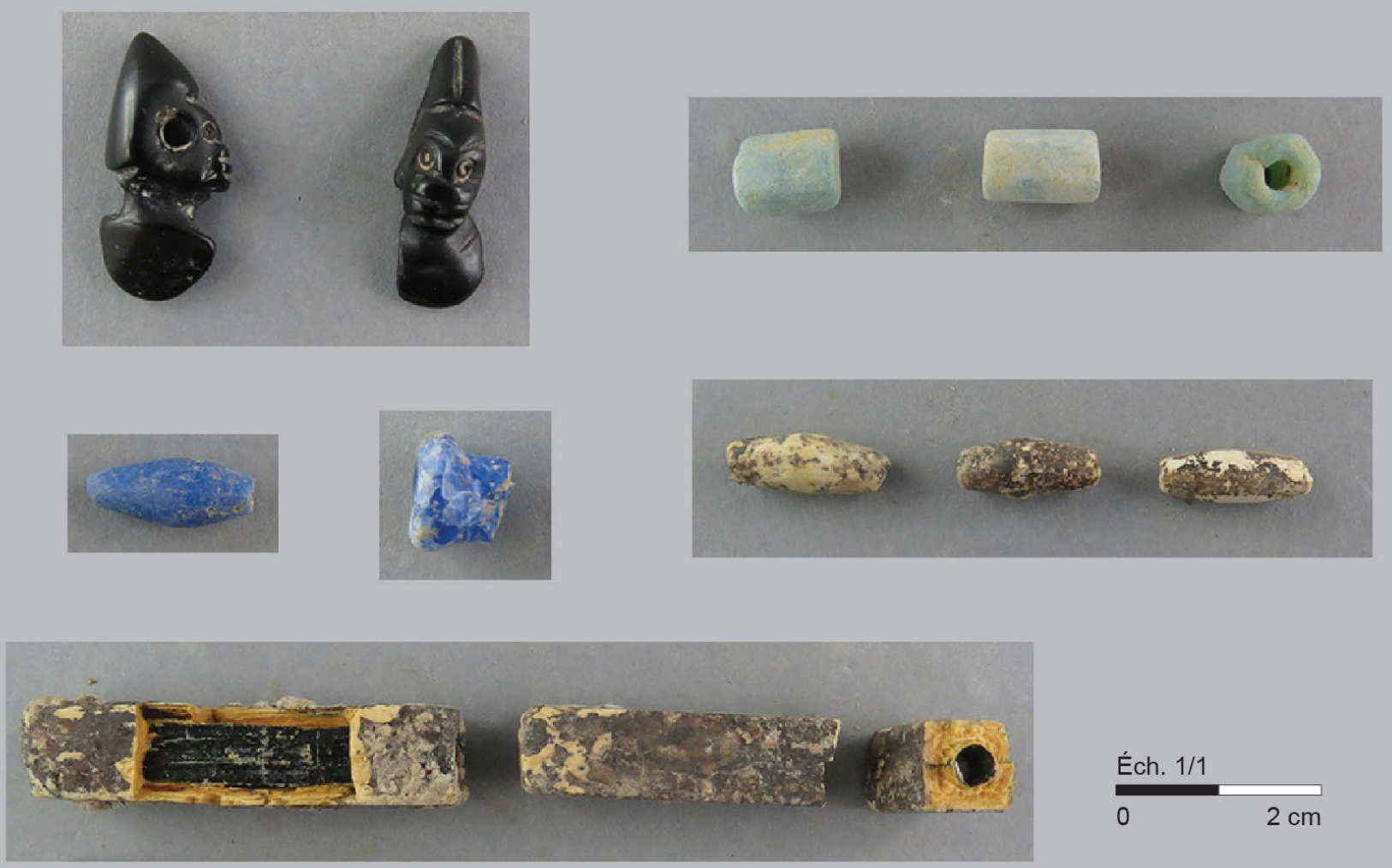

\section{Eléments de comparaison}

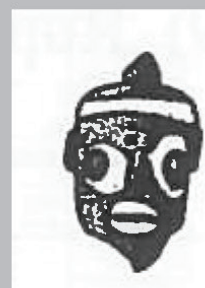

Se $B$ la

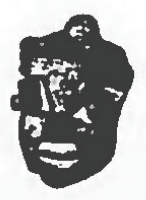

$\triangle \mathrm{Se} B \mathrm{Ib}$

Seefried, 1982

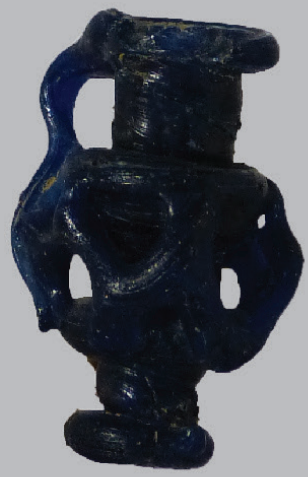

Dourges-Noyelles Godaults (62)

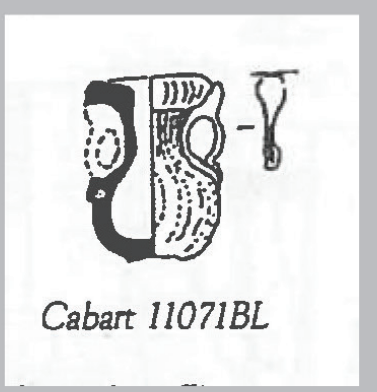

Cabart, 2003

Fig. 10 : Éléments composant le collier découvert dans les latrines 77. Photo : M.-L. Bonte. 


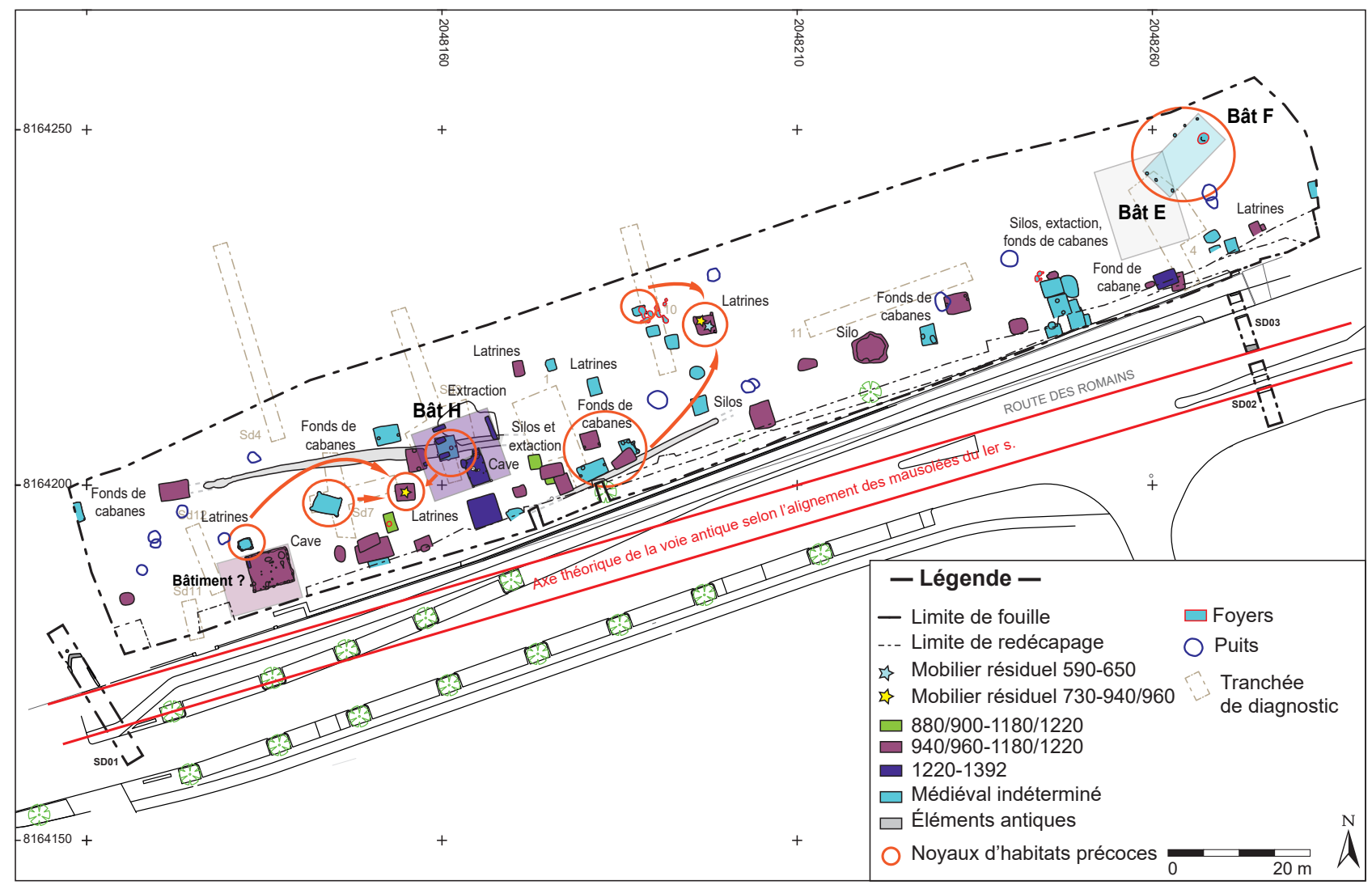

Fig. 11 : Occupations médiévales. DAO : S. Guillotin.

La nature du matériel associé aux vestiges souligne que les hommes ayant réinvesti ces lieux sont certainement les rescapés du drame. C'est ce que montre le vaisselier de facture locale associé aux vestiges, ainsi que la réalisation de pratiques bouchères identiques. L'analyse de la faune du 2, route des Romains présente cependant un indice permettant de confirmer que les habitants ont subi une crise ; il s'agit du retour de la consommation du cheval, que la tradition attribue généralement à des contextes de crise ou à la présence de troupes germaniques. Quelques restes d'équidés ont en effet été mis au jour, présentant des traces de découpe bouchère identiques à celles effectuées sur les bœufs et porcs généralement consommés sur le site. On notera qu'un phénomène identique a été observé au niveau du spectre faunique du 8-20 route des Romains [18].

\section{L'OCCUPATION MÉDIÉVALE}

La présence d'un nombre important de vestiges médiévaux a constitué une autre découverte exceptionnelle. L'unique fosse datée du XII siècle mise au jour lors de la réalisation des sondages archéologiques sur les parcelles concernées par l'opération, ne laissait en effet pas présager une telle découverte. Ces restes, au même titre que les vestiges antiques, ont été arasés ou oblitérés par les terrassements contemporains qui n'ont préservé que les nombreux aménagements en creux et, de façon partielle, les structures les moins fondées qui permettent de définir des restes de bâtiments. L'ensemble des aménagements exhumés est réparti sur l'intégralité de la surface décapée avec une plus forte concentration en bordure de la route des Romains. Ils témoignent de la présence, sur ces parcelles, d'un habitat pérenne représenté par des restes de bâtiments, deux pièces semi-excavées, de nombreux fonds de cabanes et structures d'équipement (latrines, silos, foyers, fosses d'extraction...).

Ces vestiges, et les artefacts qui leur étaient associés, permettent de mettre ces éléments en relation soit avec le village disparu d'Adelnhoffen, soit avec une portion de I'habitat dispersé de Koenigshoffen, dont les origines remontent au haut Moyen Âge. Tous deux ont été détruits en 1392 suite à une querelle entre les Strasbourgeois et leur évêque [19]. 


\section{UNE OCCUPATION DE TRANSITION?}

La découverte de fonds de cabanes présentant quatre ou plus de quatre poteaux ainsi que du matériel daté au plus tôt du VI ${ }^{\mathrm{e}}$ siècle montre que les parcelles concernées par la fouille ont certainement été occupées très tôt. La corrélation entre la localisation des anciens noyaux d'habitats du Bas-Empire, des fosses à plus de quatre poteaux et des artefacts médiévaux les plus anciens suggère que les premières occupations médiévales font suite aux dernières occupations antiques. De même, nombreux sont les aménagements médiévaux qui sont installés au-dessus ou contre les anciens éléments de parcellaire antiques, ce qui plaide également en faveur de cette hypothèse. Quoi qu'il en soit, il est difficile, dans ce contexte d'établir si cette transition s'est opérée directement, ou si le site a connu un abandon de courte durée entre les Ve et VI siècles.

\section{DÉVELOPPEMENT DE L'OCCUPATION À PARTIR DU XE SIÈCLE}

\section{L'HABITAT}

À partir du Xe siècle, I'agglomération de Koenigshoffen connaît un essor comme le montre la grande quantité de vestiges et d'artefacts mis au jour. Cette occupation est plus particulièrement constituée de deux probables bâtiments, élevés sur vides sanitaires, disposant de pièces semi-excavées qui pouvaient faire office de celliers ou de locaux de travail, associés à des latrines. Elle est complétée par de nombreux fonds de cabanes à deux poteaux axiaux ou dépourvus de poteaux, des foyers, de nombreux silos et des fosses d'extraction. L'analyse du mobilier céramique a permis de définir deux phases d'occupation situées entre le milieu du $X^{e}$ siècle et la fin du XIVe siècle. La façon dont était organisé cet habitat est peu claire, à l'exception du fait qu'il se développait en bordure de l'ancien axe antique menant aux portes de la ville de Strasbourg, connu à partir de 1406 sous le nom de Lantstrasse, correspondant à l'actuelle route des Romains. Toutefois, les mentions concernant l'agglomération de Koenigshoffen au XIV siècle font état d'un village constitué de corps de fermes non groupés [20], ce que laissent entrevoir les rares vestiges mis au jour par la fouille où seuls deux bâtiments associés à des structures d'équipement ont été observés, le reste des vestiges étant constitué de fosses de stockage et de locaux artisanaux.

[20] DOTTORI 2017, p. 263.

[21] FLOTTE 2020, p. X.

[22] KERN 1994.
D'autres indices d'occupations médiévales ont été mis au jour au 8-20 route des Romains où un silo daté du premier Moyen Âge a été découvert [21] ; au 24-26 route des Romains, une fosse avec des pots de poêle datés du XII ${ }^{\text {e }}$ siècle a également été mise au jour en 1994 par E. Kern [22]. Une présence plus marquée au XIV siècle est enregistrée sur ce site et celui du 24-26 route des Romains où ont été mises au jour des caves qui semblent reprendre les orientations des anciens bâtiments antiques comme c'est le cas pour notre site. Les caves découvertes sur ce dernier site, sous les caves des fermes du XIXe siècle, étaient construites en brique.

\section{DES ACTIVITÉS ARTISANALES ET AGRICOLES}

Le site a livré un grand nombre de fonds de cabanes et de silos confirmant le caractère rural de cet habitat, tourné vers des activités agro-pastorales et un artisanat dévolu à l'autonomie des différentes cellules d'habitat plutôt qu'à une économie de marché.

Un nombre important de scories (plus de $47 \mathrm{~kg}$ ) a été mis au jour dans le comblement de fonds de cabanes, latrines et fosses d'extraction, plus particulièrement à I'ouest de la fouille. Ces restes matériels indiquent la pratique d'activités tournées vers la métallurgie, même si aucune structure de production n'a été retrouvée dans l'emprise de la fouille; des restes de parois de fours et de scories réduites signalent toutefois la présence d'infrastructures dans un secteur proche, ces dernières ayant pu être oblitérées par les installations ou terrassements contemporains.

L'analyse des restes détritiques de faune découverts dans certains fonds de cabane, latrines et silos permet également d'établir la pratique d'activités bouchères. Le faciès de l'alimentation carnée est différent de celui observé durant l'Antiquité. On distingue en effet une forte représentativité des équidés, lesquels présentent des traces d'équarrissage, et une moins nette importance des caprinés. L'alimentation de base est assurée par les bovins et les suinés dont l'âge d'abattage est plus avancé que celui des animaux antiques. Pour ces deux espèces, on note une surreprésentation des bas de pattes que l'on peut considérer comme des rejets primaires de boucherie.

\section{LES VESTIGES CONTEMPORAINS}

Suite au démantèlement des villages de Koenigshoffen et d'Adelnhoffen en 1392, les deux agglomérations ne seront pas reconstruites, car considérées comme trop proches des fortifications de la ville de Strasbourg, donc pouvant servir de point d'appui à des sièges 


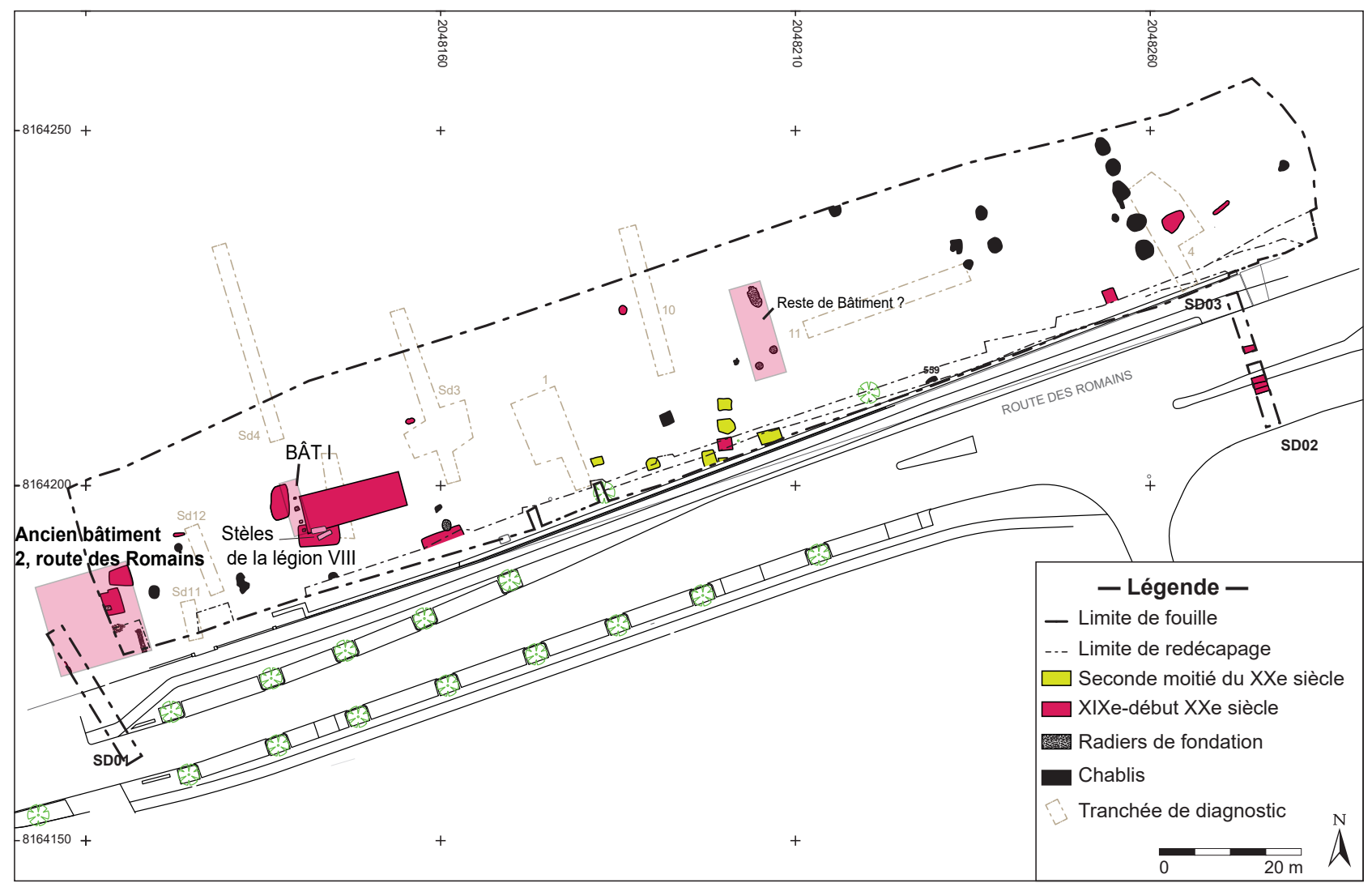

Fig. 12 : Les vestiges et restes contemporains. DAO : S. Guillotin.

répétés à l'encontre de la ville. Ces anciens terroirs et fermages sont donc transformés en champs, comme le mentionne un recollement des biens du couvent de la Chartreuse et comme l'illustrent les cartes les plus anciennes représentant Strasbourg et ses abords occidentaux.

Ces parcelles laniérées dévolues à l'agriculture et aux vergers sont par conséquent déclarées non constructibles suite à l'ordonnance du 9 décembre 1793, car situées dans le périmètre des fortifications.

De ce fait, la reconstruction de Koenigshoffen, sous la forme d'un faubourg de la ville de Strasbourg, n'intervient pas avant le milieu du XIX siècle et elle est, dans un premier temps, liée au développement puis à I'essor des brasseries. Mais le secteur impacté par la fouille ne faisait pas encore, à ce moment-là, l'objet de reconstruction, étant donné sa localisation dans les secteurs militarisés.

Lors de la fouille, plusieurs structures ont toutefois été attribuées à l'époque contemporaine, soit en raison de la présence de matériel dans leur comblement, soit compte tenu des relations stratigraphiques qu'elles entretenaient avec des installations médiévales. Ces restes sont de deux natures : on distingue d'une part des fosses de grand module, très profondes, dont le profil évoque des fosses d'extraction, et d'autre part des radiers de fondations et des murs en dur en lien avec des bâtiments disparus. Les fosses dites d'extraction ont été mises au jour à l'ouest de la fouille. C'est dans l'une d'elles qu'ont été découvertes les deux stèles de légionnaires, associées à une semelle cloutée de chaussure dont la facture allemande permet une datation de la fin du XIXe siècle. Il est possible que ce secteur non constructible, situé à la sortie du premier noyau construit de Koenigshoffen, ait servi de lieu d'extraction pour la construction de nouveaux corps de fermes à pans de bois.

Les restes de bâtiments sont représentés par deux types d'architectures différents : on distingue en premier lieu les restes de fondations en dur correspondant au corps de ferme du 2, route des Romains, dont la construction remonte au début du XXe siècle et qui a été démoli en 2013 dans le cadre des projets d'aménagement du tramway. Les autres aménagements concernent des radiers de fondations remplis de gravier et de sable dont certains formaient encore des alignements, et qui devaient accueillir des plots en grès soutenant l'armature de bâtiments hors sol. La relation stratigraphique observée entre l'un des radiers de fondation et la fosse qui recelait les stèles, a permis d'établir que ce bâtiment ne pouvait être daté qu'à partir du début du $X X^{e}$ siècle. 
Les recherches menées dans les archives de la ville ont permis de définir l'existence de bâtiments à pans de bois, démontables dans le rayon d'action des fortifications à I'Elsau ou encore à Cronenbourg. Ces édifices, établis en secteur militarisé devaient être facilement démontables en cas de siège. Pour exemple, on peut citer l'église à pans de bois St Arbogast, établie à I'Elsau en 1910, dans le rayon d'action des fortifications, et encore en élévation de nos jours.

Aucun indice ne nous est parvenu quant à la fonction de ces bâtiments. L'hypothèse la plus vraisemblable reste toutefois que ces maisons ont pu être intégrées à une sorte de no man's land où étaient logés de façon peu salubre des ouvriers ou manœuvres embauchés à bas coût dans le cadre du développement économique de Strasbourg et du quartier de Koenigshoffen. Le cadre illicite de ces logements pourrait expliquer le caractère dérangeant de la découverte des stèles de légionnaires, qu'on a préféré ré-enfouir plutôt que de rediriger vers la Société pour la conservation des monuments historiques d'Alsace créée en 1855 . On appréciera ici le caractère burlesque de la situation : à savoir, le ré-enfouissement de ces stèles, laissant la joie aux archéologues du XXI siècle d'exhumer pour la dernière fois ces blocs. Ces derniers ont finalement été transférés au Musée archéologique de la Ville de Strasbourg, où ils sont désormais conservés!

\section{CONCLUSION}

La fouille réalisée au 2, route des Romains a permis d'enrichir considérablement les connaissances sur le devenir des parcelles situées à l'entrée de Koenigshoffen de l'époque antique à nos jours. Outre I'occupation double du Haut-Empire (continuité de la nécropole et de I'habitat), qui était attendue à l'issue des deux diagnostics réalisés sur l'emprise des travaux en 2014, les fouilles ont permis de dégager et de documenter les restes de plusieurs habitats inédits datés de la fin de I'Antiquité, de la période médiévale et du début de le première occupation allemande. On a ainsi pu observer pour la première fois à Koenigshoffen une continuité de I'habitat antique entre le début du second tiers du II siècle et le $V^{\mathrm{e}}$ siècle, au travers d'une réoccupation des lieux après le déclin de l'agglomération. Outres ces questions relatives au développement puis à la régression du noyau urbain, la fouille a permis de compléter nos connaissances relatives à l'artisanat potier, d'établir un lien avec des sites établis intégrés à la ceinture d'eau de la ville en se penchant sur la distribution des productions élaborées dans ces ateliers, et plus important encore, de définir sur ce site l'existence d'une dimension cultuelle et culturelle entretenue par les artisans.
Les quelques restes, certes lacunaires, attribuables à la nécropole militaire du I ${ }^{\text {er }}$ siècle, ont également permis d'ouvrir quelques pistes de réflexion quant au développement de cette dernière, mais également de redécouvrir deux stèles de légionnaires en grès mentionnant pour la première fois les noms de légionnaires de la VIII légion, monuments qui auraient dû trouver leur place dans les collections de la ville dès la fin du XIXe siècle.

Cette opération préventive a également contribué à mettre au jour une portion d'habitat correspondant au Koenigshoffen médiéval ou au village disparu d'Adelnhoffen, dont on ne connaissait à ce jour que des mentions écrites provenant de sources anciennes [23].

Enfin, et contre toute attente, les recherches effectuées sous le terrain de I'ASPTT ont montré que ces parcelles situées dans le second périmètre des fortifications de la ville, que I'on croyait restées à l'état de champs et de vergers jusqu'à la mise en place du stade, étaient occupées, au début du XXe siècle, par des bâtiments à pans de bois dont le souvenir n'a pas perduré jusqu'à nous et qui ne figuraient sur aucun des plans élaborés durant l'occupation allemande.

Pour conclure, on peut mentionner que les fouilles récentes ont permis d'offrir une nouvelle vision du passé sur le temps long de la partie est de la route des Romains, et ce, malgré les nombreuses pertes d'informations relatives à la création de la plaine sportive à la fin des années 1960.

[23] Нимм 1971, p. 75. 
Baudoux, Juliette \& Cicutra, Heidi, 2017, «L'activité potière à Koenigshoffen », dans Bernadette Schnitzler \& Pascal Flotté (éd.), Koenigshoffen, époque romaine. Un quartier civil de Strasbourg-Argentorate du Ir-IVe siècle après J.-C., Strasbourg, p. 106-116.

Beraux, Marianne \& Pichot, Adeline, 2021, «Deux nouvelles inscriptions de militaires de la légion VIII Augusta (2, route des Romains, Strasbourg-Koenigshoffen) », Revue Archimède 8.

Blin, Séverine \& Flotte, Pascal, 2017, «La nécropole du Ier au début du II siècle après J.-C. », dans Bernadette Schnitzler \& Pascal Flotté (éd.), Koenigshoffen, époque romaine. Un quartier civil de Strasbourg-Argentorate du I'r-IVe siècle après J.-C., Strasbourg, p. 174-179.

DotrorI, Boris, 2017, «Dum Kunigeshoven fuit villa : Koenigshoffen à la période médiévale (XII $-\mathrm{XV}$ e siècle) 》, dans Bernadette Schnitzler \& Pascal Flotté (éd.), Koenigshoffen, époque romaine. Un quartier civil de Strasbourg-Argentorate du Ir-IVe siècle après J.-C., p. 263-265.

FABBRo, Morgane, 2020, «Alimentation carnée à Koenigshoffen antique. Données archéozoologiques 2, route des Romains, Strasbourg, Alsace », Master 2, Archéologie de l'Europe moyenne, Université de Strasbourg.

FLotre et al., 2020, «Strasbourg, Bas-Rhin, 8 - 20 route des Romains. La nécropole de Strasbourg-Koenigshoffen du $1^{\text {er }}$ siècle apr. J.-C. au début du 2e siècle », Rapport final d'opération d'archéologie préventive, Archéologie-Alsace, Sélestat. Gervreaud, Jean-Baptiste \& Bebien, Cécile, 2014, « Strasbourg, Bas-Rhin, 2 route des Romains », Rapport de diagnostic, Archéologie-Alsace, Sélestat.

Gohrer, Pauline \& Murer, Axelle, 2020, « Les fours de potier et la céramique du 2, route des Romains (StrasbourgKoenigshoffen) : nouvelles données sur l'artisanat antique » dans Société Française d'Étude de la Céramique Antique en Gaule, Actes du Congrès de Lyon, 24-27 septembre 2020, p. 593-612.

Нuмm, André, 1971, Villages et hameaux disparus en Basse-Alsace. Contribution à I'histoire de I'habitat rural (XII - -XVIII $I^{e}$ siècles), Strasbourg.

Kern, Erwin, 1994, « Strasbourg-Koenigshoffen, 24-26, route des Romains - Le Capitole », Inventaire des objets dévolus au propriétaire du terrain, SRA Alsace, DRAC Strasbourg.

Murer, Axelle et al., 2013, « Brumath, 8 rue du Collège, un nouvel éclairage sur le quartier des potiers, Alsace, Bas-Rhin (67) », Rapport final d'opération d'archéologie préventive, ANTEA-Archéologie, SRA Grand Est, Habsheim.

Murer, Axelle et al., 2016, «Strasbourg Hôpital Civil, Alsace, Bas-Rhin (67) », Rapport final d'opération d'archéologie préventive, ANTEA-Archéologie, SRA Grand Est, Habsheim.

Murer, Axelle et al., 2020, «Strasbourg-Koenigshoffen. Extension du Tramway - 2, route des Romains, Alsace, Bas-Rhin (67) », Rapport final d'opération d'archéologie préventive, ANTEA- Archéologie, SRA Grand Est, Habsheim.

Nilles, Richard \& Cicutra, Heidi, 2014, « Alsace-Bas-Rhin, Strasbourg- Koenigshoffen, 2-20 route des Romains. Données complémentaires sur l'occupation antique extrême orientale du vicus de Koenigshoffen », Rapport de diagnostic archéologique, INRAP Grand Est, Strasbourg.

Schnitzler, Bernadette, 2017, «La nécropole gallo-romaine de la Porte Blanche », dans Bernadette Schnitzler \& Pascal Flotté (éd.), Koenigshoffen, époque romaine. Un quartier civil de Strasbourg-Argentorate du Ier-IVe siècle après J.-C., Strasbourg, p. 230-234.

Schnitzler, Bernadette, 2017, «Le Haut Moyen Âge : une période encore mal connue », dans Bernadette Schnitzler \& Pascal Flotté (éd.), Koenigshoffen, époque romaine. Un quartier civil de Strasbourg-Argentorate du Ier-IVe siècle après J. -C., Strasbourg, p. 258-262.

Schnitzler, Bernadette \& Flotte, Pascal (dir.), 2017, Koenigshoffen, époque romaine. Un quartier civil de Strasbourg-

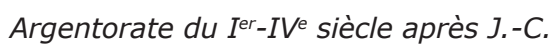

Waton, Marie-Dominique \& Flotte, Pascal, 2017, « Voirie et organisation de l'habitat dans le vicus des canabae », dans Bernadette Schnitzler \& Pascal Flotté (éd.), Koenigshoffen, époque romaine. Un quartier civil de de Strasbourg-Argentorate du Ier-IVe siècle après J.-C., p. 89-93.

Willems, Sonja \& Favennec, Benoît, 2019, « Abandon et clôture des fours de potiers : un geste systématique ? », dans Société Française d'Étude de la Céramique Antique en Gaule, Actes du Congrès de Maubeuge- Bavay, 30 mai-2 juin 2019, p. 177-192. 